Supporting Information

\title{
Fluorine...Fluorine Interactions in a High-Pressure Layered Phase of Perfluorobenzene
}

\author{
Michalina Rusek $^{1}$, Karolina Kwaśna ${ }^{1}$, Armand Budzianowski ${ }^{2}$ and \\ Andrzej Katrusiak ${ }^{1}$
}

${ }^{1}$ Faculty of Chemistry, Adam Mickiewicz University, Umultowska 89b , 61-614 Poznań, Poland.

${ }^{2}$ National Centre for Nuclear Research, Sołtana 7, 05-400 Otwock, Poland.

Table S1. Crystal data and structure-refinements details of $\mathrm{C}_{6} \mathrm{~F}_{6}$ phase I at 0.31 $\mathrm{GPa} / 296 \mathrm{~K}$ and $0.60 \mathrm{GPa} / 296 \mathrm{~K}$.

\begin{tabular}{|c|c|c|}
\hline Pressure & $0.31(2) \mathrm{GPa}$ & $0.60(2) \mathrm{GPa}$ \\
\hline Temperature (K) & $296(2)$ & $296(2)$ \\
\hline Formula weight & 186.06 & 186.06 \\
\hline Wavelength $(\AA)$ & 0.71073 & 0.71073 \\
\hline Crystal system & Monoclinic & Monoclinic \\
\hline Space group & $P 2_{1} / n$ & $P 2_{1} / n$ \\
\hline \multicolumn{3}{|l|}{ Unit cell dimensions $\left(\AA,{ }^{\circ}\right)$} \\
\hline$a$ & $5.9567(5)$ & $5.9457(11)$ \\
\hline$b$ & $9.535(3)$ & $9.423(7)$ \\
\hline$c$ & $16.963(5)$ & $16.8697(18)$ \\
\hline$\beta$ & $94.078(11)$ & $94.173(11)$ \\
\hline Volume $\left(\AA^{3}\right)$ & $961.0(4)$ & $942.6(7)$ \\
\hline$Z$ & 6 & 6 \\
\hline Calculated density $\left(\mathrm{g} / \mathrm{cm}^{3}\right)$ & 1.929 & 1.967 \\
\hline Absorption coeff. $\left(\mathrm{mm}^{-1}\right)$ & 0.236 & 0.241 \\
\hline $\mathrm{F}(000)$ & 540 & 540 \\
\hline Crystal size (mm) & $0.47 / 0.39 / 0.30$ & $0.47 / 0.39 / 0.30$ \\
\hline $\begin{array}{l}\theta \text {-range for data collection } \\
\left({ }^{\circ}\right)\end{array}$ & 3.55 to 27.12 & 4.06 to 27.33 \\
\hline Min/max indices: $h, k, l$ & $-7 / 7,-8 / 8,-16 / 16$ & $-7 / 6,-5 / 5,-20 / 21$ \\
\hline Reflect. Collected/unique & $3117 / 500$ & $2991 / 475$ \\
\hline $\mathrm{R}_{\text {int }}$ & 0.0317 & 0.0324 \\
\hline Refinement method & \multicolumn{2}{|c|}{ Full-matrix least-squares on $\mathrm{F}^{2}$} \\
\hline Completeness (\%) & 25.7 & 22.2 \\
\hline Data/restrains/parameters & $500 / 66 / 164$ & $475 / 63 / 164$ \\
\hline Goodness-of-fit on $\mathrm{F}^{2}$ & 1.338 & 1.205 \\
\hline Final $R_{1} / w_{2}\left(I>2 \sigma_{1}\right)$ & $0.0794 / 0.2433$ & $0.0701 / 0.2107$ \\
\hline $\mathrm{R}_{1} / \mathrm{wR}_{2}$ (all data) & $0.0932 / 0.2740$ & $0.0821 / 0.2392$ \\
\hline
\end{tabular}


Table S2. Crystal data and structure-refinements details of $\mathrm{C}_{6} \mathrm{~F}_{6}$ phase II at 0.89 $\mathrm{GPa} / 296 \mathrm{~K}, 1.00 \mathrm{GPa} / 296 \mathrm{~K}$ and $1.07 \mathrm{GPa} / 296 \mathrm{~K}$.

\begin{tabular}{|c|c|c|c|}
\hline Pressure & $0.89(2) \mathrm{GPa}$ & $1.00(2) \mathrm{GPa}$ & $1.07(2) \mathrm{GPa}$ \\
\hline Temperature $(\mathrm{K})$ & $296(2)$ & $296(2)$ & $296(2)$ \\
\hline Formula weight & 186.06 & 186.06 & 186.06 \\
\hline Wavelength ( & 0.71073 & 0.71073 & 0.71073 \\
\hline Crystal system & Monoclinic & Monoclinic & Monoclinic \\
\hline Space group & $C 2 / c$ & $C 2 / c$ & $C 2 / c$ \\
\hline \multicolumn{4}{|l|}{ Unit cell dimensions $\left(\AA \mathrm{A}^{\circ}\right)$} \\
\hline$a$ & $12.879(2)$ & $12.866(7)$ & $12.851(4)$ \\
\hline$b$ & $7.383(3)$ & $7.360(7)$ & $7.349(4)$ \\
\hline$c$ & $8.449(1)$ & $8.423(2)$ & $8.3940(12)$ \\
\hline$\beta$ & $135.94(2)$ & $136.14(3)$ & $136.133(19)$ \\
\hline Volume $\left(\AA^{3}\right)$ & $558.7(2)$ & $552.7(6)$ & $549.4(4)$ \\
\hline$Z$ & 4 & 4 & 4 \\
\hline Calculated density $\left(\mathrm{g} / \mathrm{cm}^{3}\right)$ & 2.212 & 2.236 & 2.250 \\
\hline Absorption coeff. $\left(\mathrm{mm}^{-1}\right)$ & 0.271 & 0.274 & 0.275 \\
\hline$F(000)$ & 360 & 360 & 360 \\
\hline Crystal size (mm) & $0.28 / 0.33 / 0.31$ & $0.28 / 0.33 / 0.31$ & $0.28 / 0.33 / 0.31$ \\
\hline$\theta$-range data collection $\left(^{\circ}\right)$ & 4.844 to 23.554 & 3.565 to 25.662 & 3.594 to 26.016 \\
\hline Min/max indices: $h, k, l$ & $\begin{array}{l}-14 / 14,-6 / 6,- \\
10 / 10\end{array}$ & $\begin{array}{l}-13 / 14,-6 / 6,- \\
10 / 10\end{array}$ & $\begin{array}{l}-14 / 14,-6 / 6,- \\
10 / 10\end{array}$ \\
\hline Reflect. Collected/unique & $938 / 144$ & $956 / 152$ & $937 / 146$ \\
\hline $\mathrm{R}_{\text {int }}$ & 0.0296 & 0.0433 & 0.0413 \\
\hline Refinement method & \multicolumn{3}{|c|}{ Full-matrix least-squares on $\mathrm{F}^{2}$} \\
\hline Completeness (\%) & 25.7 & 25.9 & 26.7 \\
\hline Data/restrains/parameters & $144 / 6 / 55$ & $152 / 6 / 55$ & $146 / 6 / 55$ \\
\hline Goodness-of-fit on $\mathrm{F}^{2}$ & 1.191 & 1.230 & 1.196 \\
\hline Final $R_{1} / w R_{2}\left(I>2 \sigma_{1}\right)$ & $0.0192 / 0.0318$ & $0.0282 / 0.0682$ & $0.0272 / 0.0683$ \\
\hline $\mathrm{R}_{1} / \mathrm{w} \mathrm{R}_{2}$ (all data) & $0.0267 / 0.0348$ & $0.0350 / 0.0730$ & $0.0302 / 0.0712$ \\
\hline
\end{tabular}


Table S3. Crystal data and structure-refinements details of $\mathrm{C}_{6} \mathrm{~F}_{6}$ phase II at 0.31 $\mathrm{GPa} / 296 \mathrm{~K}$ and $0.60 \mathrm{GPa} / 296 \mathrm{~K}$.

\begin{tabular}{|c|c|c|}
\hline Pressure & $1.55(2)$ & $1.90(2) \mathrm{GPa}$ \\
\hline Temperature (K) & $296(2)$ & $296(2)$ \\
\hline Formula weight & 186.06 & 186.06 \\
\hline Wavelength $(\AA)$ & 0.71073 & 0.71073 \\
\hline Crystal system & Monoclinic & Monoclinic \\
\hline Space group & $C 2 / c$ & $C 2 / c$ \\
\hline \multicolumn{3}{|l|}{ Unit cell dimensions $\left(\AA,{ }^{\circ}\right)$} \\
\hline$a$ & $12.720(3)$ & $12.630(5)$ \\
\hline$b$ & $7.293(3)$ & $7.260(5)$ \\
\hline$c$ & $8.2370(10)$ & $8.1461(14)$ \\
\hline$\beta$ & $136.252(16)$ & $136.34(2)$ \\
\hline Volume $\left(\AA^{3}\right)$ & $528.4(3)$ & $515.7(4)$ \\
\hline$Z$ & 4 & 4 \\
\hline Calculated density $\left(\mathrm{g} / \mathrm{cm}^{3}\right)$ & 2.339 & 2.397 \\
\hline Absorption coeff. $\left(\mathrm{mm}^{-1}\right)$ & 0.286 & 0.293 \\
\hline $\mathrm{F}(000)$ & 360 & 360 \\
\hline Crystal size (mm) & $0.28 / 0.33 / 0.31$ & $0.28 / 0.33 / 0.31$ \\
\hline $\begin{array}{l}\theta \text {-range for data collection } \\
\left(^{\circ}\right)\end{array}$ & 3.629 to 26.328 & 3.652 to 26.653 \\
\hline Min/max indices: $h, k, l$ & $-14 / 14,-6 / 6,-10 / 10$ & $-13 / 13,-6 / 6,-10 / 10$ \\
\hline Reflect. Collected/unique & $804 / 126$ & $758 / 116$ \\
\hline $\mathrm{R}_{\text {int }}$ & 0.0364 & 0.0260 \\
\hline Refinement method & \multicolumn{2}{|c|}{ Full-matrix least-squares on $\mathrm{F}^{2}$} \\
\hline Completeness (\%) & 23.4 & 21.2 \\
\hline Data/restrains/parameters & $126 / 0 / 55$ & $116 / 18 / 55$ \\
\hline Goodness-of-fit on $\mathrm{F}^{2}$ & 1.005 & 1.196 \\
\hline Final $R_{1} / w_{2}\left(I>2 \sigma_{1}\right)$ & $0.0143 / 0.0260$ & $0.0208 / 0.0392$ \\
\hline $\mathrm{R}_{1} / \mathrm{wR}_{2}$ (all data) & $0.0185 / 0.0269$ & $0.0244 / 0.0401$ \\
\hline
\end{tabular}


Table S4. Crystal data and structure-refinements details of $\mathrm{C}_{6} \mathrm{~F}_{6}$ phase I at low-temperature conditions.

\begin{tabular}{|c|c|c|c|}
\hline Temperature $(\mathrm{K})$ & $106(2)$ & $132(2)$ & $183(2)$ \\
\hline Formula weight & 186.06 & 186.06 & 186.06 \\
\hline Wavelength $(\AA)$ & 1.54184 & 1.54184 & 1.54184 \\
\hline Crystal system & Monoclinic & Monoclinic & Monoclinic \\
\hline Space group & $P 2_{1} / n$ & $P 2_{1} / n$ & $P 2_{1} / n$ \\
\hline $\begin{array}{l}\text { Unit cell dimensions }\left(\AA^{\circ},{ }^{\circ}\right) \\
\text { a } \\
\mathrm{b} \\
\mathrm{c} \\
\beta \\
\text { Volume }\left(\AA^{3}\right)\end{array}$ & $\begin{array}{l}5.9102(2) \\
9.0839(3) 16.7901(6) \\
93.956(3) \\
899.27(5)\end{array}$ & $\begin{array}{l}5.91354(18) \\
9.1308(3) 16.7933(5) \\
94.003(3) \\
904.55(5)\end{array}$ & $\begin{array}{l}5.9371(4) \\
9.2879(5) \\
16.8314(14) \\
94.035(8) \\
925.83(12)\end{array}$ \\
\hline Calculated density $\left(\mathrm{g} / \mathrm{cm}^{3}\right)$ & 2.061 & 2.049 & 2.002 \\
\hline Absorption coefficient $\left(\mathrm{mm}^{-1}\right)$ & 2.352 & 2.347 & 2.289 \\
\hline Crystal size (mm) & $5.2 / 0.5 / 0.5$ & $5.2 / 0.5 / 0.5$ & $5.2 / 0.5 / 0.5$ \\
\hline$\theta$-range for data collection $\left({ }^{\circ}\right)$ & 5.282 to 71.326 & 5.281 to 71.341 & 5.269 to 71.042 \\
\hline Min/max indices:h, $\mathrm{k}, 1$ & $-7 / 7,-10 / 11,-20 / 20$ & $-7 / 7,-10 / 11,-20 / 20$ & $-7 / 4,-11 / 11,-20 / 19$ \\
\hline $\begin{array}{l}\text { Reflect. Collected/unique } \\
\mathrm{R}_{\text {int }} \\
\text { Refinement method }\end{array}$ & $\begin{array}{l}12900 / 1735 \\
0.1563\end{array}$ & $\begin{array}{l}12631 / 1700 \\
0.1847 \\
\text { I-matrix least-squares or }\end{array}$ & $\begin{array}{l}5245 / 1706 \\
0.1457 \\
\text { F2 }\end{array}$ \\
\hline Completeness (\%) & 100.0 & 100.0 & 96.9 \\
\hline Data / restraints / parameters & $1735 / 0 / 163$ & $1750 / 0 / 163$ & $1706 / 0 / 164$ \\
\hline GooF on $\mathrm{F}^{2}$ & 1.070 & 1.083 & 1.278 \\
\hline Final $R_{1} / w R_{2}\left(I>2 \sigma_{1}\right)$ & $0.0523 / 0.1498$ & $0.0618 / 0.1646$ & $0.0962 / 0.2387$ \\
\hline $\mathrm{R}_{1} / \mathrm{wR}_{2}$ (all data) & $0.0601 / 0.1598$ & $0.0687 / 0.1769$ & $0.1257 / 0.2770$ \\
\hline
\end{tabular}


Table S5. Crystal data and structure-refinements details of $\mathrm{C}_{6} \mathrm{~F}_{6}$ phase I at low-temperature conditions.

\begin{tabular}{|c|c|c|c|}
\hline Temperature $(\mathrm{K})$ & $228.0(7)$ & $255.00(14)$ & 268.00(14) \\
\hline Formula weight & 186.06 & 186.06 & 186.06 \\
\hline Wavelength (A) & 1.54184 & 1.54184 & 1.54184 \\
\hline Crystal system & Monoclinic & Monoclinic & Monoclinic \\
\hline Space group & $P 2_{1} / n$ & $P 2_{1} / n$ & $P 2_{1} / n$ \\
\hline \multicolumn{4}{|l|}{ Unit cell dimensions $\left(\AA,^{\circ}\right)$} \\
\hline$a$ & $5.9568(8)$ & $5.9687(3)$ & $5.9705(3)$ \\
\hline$b$ & $9.4339(12)$ & $9.4897(4)$ & $9.5169(5)$ \\
\hline$c$ & $16.857(3)$ & $16.9317(8)$ & $16.9580(10)$ \\
\hline$\beta$ & $94.025(14)$ & $94.193(5)$ & $94.148(5)$ \\
\hline Volume $\left(\AA^{3}\right)$ & $945.0(2)$ & $956.47(8)$ & $961.04(9)$ \\
\hline Calculated density $\left(\mathrm{g} / \mathrm{cm}^{3}\right)$ & 1.962 & 1.938 & 1.929 \\
\hline Absorption coefficient $\left(\mathrm{mm}^{-1}\right)$ & 2.230 & 2.216 & 2.202 \\
\hline Crystal size (mm) & $5.2 / 0.5 / 0.5$ & $2.5 / 0.3 / 0.3$ & $2.5 / 0.3 / 0.3$ \\
\hline$\theta$-range for data collection $\left(^{\circ}\right)$ & 5.261 to 41.438 & 5.239 to 71.142 & 5.230 to 71.088 \\
\hline Min/max indices: $h, k, l$ & $-4 / 2,-4 / 8,-13 / 14$ & $-7 / 6,-11 / 11,-18 / 20$ & $-7 / 7,-11 / 11,-20 / 20$ \\
\hline Reflect. Collected/unique & $752 / 544$ & $11821 / 1858$ & $12186 / 1862$ \\
\hline $\mathrm{R}_{\text {int }}$ & 0.0547 & 0.0923 & 0.1103 \\
\hline Refinement method & \multicolumn{3}{|c|}{ Full-matrix least-squares on $\mathrm{F}^{2}$} \\
\hline Completeness (\%) & 87.3 & 99.9 & 100 \\
\hline Data / restraints / parameters & $544 / 0 / 164$ & $1858 / 0 / 163$ & $1862 / 0 / 163$ \\
\hline GooF on $\mathrm{F}^{2}$ & 1.33 & 1.119 & 1.120 \\
\hline Final $R_{1} / w R_{2}\left(I>2 \sigma_{1}\right)$ & $0.0695 / 0.1794$ & $0.0912 / 0.2934$ & $0.0940 / 0.2972$ \\
\hline $\mathrm{R}_{1} / \mathrm{wR}_{2}$ (all data) & $0.0890 / 0.2104$ & $0.1041 / 0.3178$ & $0.1166 / 0.3392$ \\
\hline
\end{tabular}
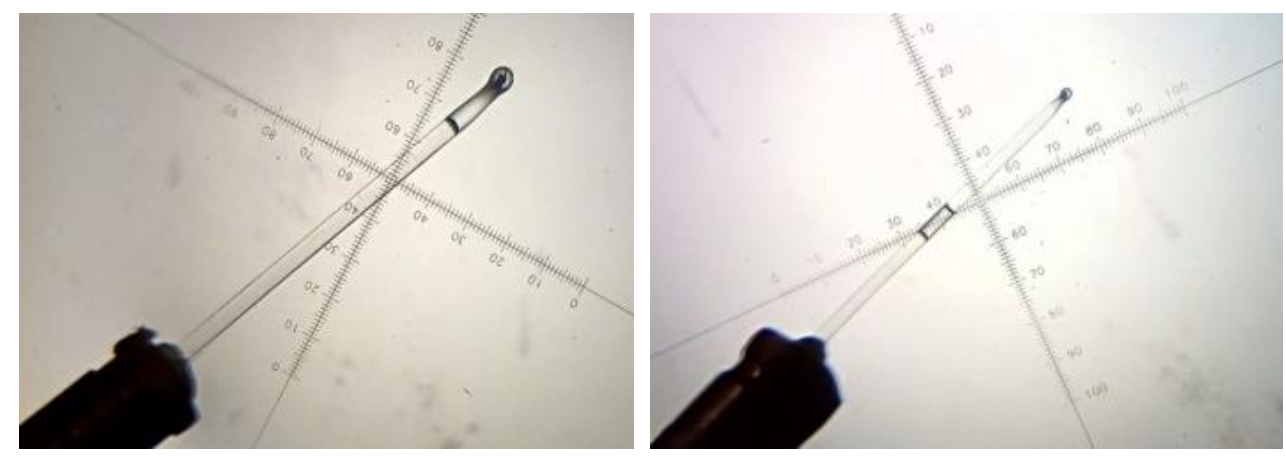

Figure S1. Both sides enclosed capillaries $0.5 \mathrm{~mm}$ (left) and $0.3 \mathrm{~mm}$ (right) with liquid perfluorobenezne inside. 


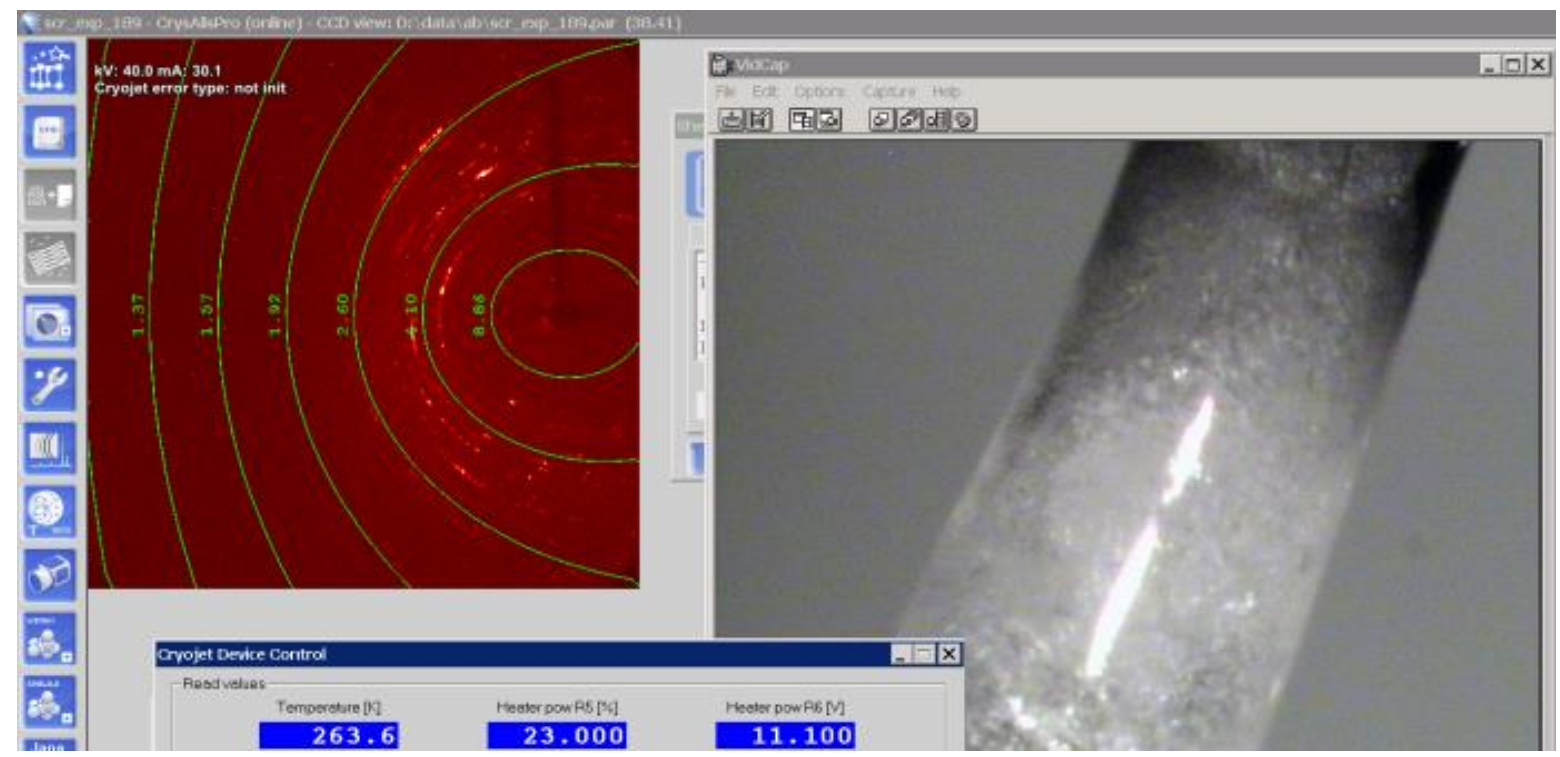

Figure S2. A powder solid state of perfluorobenezne obtained on the diffractometer by in-situ frozen inside of $0.5 \mathrm{~mm}$ capillary. On the left are well visible powder diffractions rings.

Table S6. Short contacts C-F $\cdots F^{\prime}-C^{\prime}$ and their F $\cdots F^{\prime}$ distance: C-F $\cdots F^{\prime}$ angle/ $C^{\prime}-F^{\prime} \cdots F$ angle and $\mathrm{F} \cdots \mathrm{F}^{\prime}$ distance in phase $\mathrm{I}$ at 0.31 and $0.60 \mathrm{GPa}$.

\begin{tabular}{llllll}
\hline & $0.31 \mathrm{GPa}$ & & $0.60 \mathrm{GPa}$ & \multicolumn{1}{l}{ Symmetry code } \\
\hline $\mathrm{F} 6 \cdots \mathrm{F} 7$ & $170.73^{\circ} / 139.72^{\circ}$ & $2.868(11) \AA$ & $172.96^{\circ} / 136.66^{\circ}$ & $2.834(11) \AA$ & $-1+\mathrm{x}, \mathrm{y}, \mathrm{z}$ \\
$\mathrm{F} 1 \cdots \mathrm{F} 8$ & $111.66^{\circ} / 104.40^{\circ}$ & $2.954(16) \AA$ & $115.23^{\circ} / 102.09^{\circ}$ & $2.938(18) \AA$ & $1-\mathrm{x},-\mathrm{y}, 1-\mathrm{z}$ \\
$\mathrm{F} 3 \cdots \mathrm{F} 6$ & $88.67^{\circ} / 86.15^{\circ}$ & $2.939(14) \AA$ & $86.68^{\circ} / 81.56^{\circ}$ & $3.017(17) \AA$ & $1+\mathrm{x}, \mathrm{y}, \mathrm{z}$ \\
$\mathrm{F} 2 \cdots \mathrm{F} 4$ & $108.44^{\circ} / 141.89^{\circ}$ & $3.039(15) \AA$ & $111.33^{\circ} / 141.16^{\circ}$ & $2.954(17) \AA$ & $1.5-\mathrm{x},-0.5+\mathrm{y}, 1.5-\mathrm{z}$ \\
$\mathrm{F} 7 \cdots \mathrm{F} 1$ & $99.84^{\circ} / 132.47^{\circ}$ & $3.098(23) \AA$ & $98.47^{\circ} / 138.51^{\circ}$ & $3.031(15) \AA$ & $1-\mathrm{x},-\mathrm{y}, 1-\mathrm{z}$ \\
$\mathrm{F} 1 \cdots \mathrm{F} 2$ & $106.56^{\circ} / 95.18^{\circ}$ & $3.111(24) \AA$ & $103.93^{\circ} / 87.71^{\circ}$ & $3.134(22) \AA$ & $1-\mathrm{x},-\mathrm{y}, 1-\mathrm{z}$ \\
\hline
\end{tabular}

Table S7. Short contacts C-F $\cdots F^{\prime}-C^{\prime}$ and their F $\cdots F^{\prime}$ distance: C-F $\cdots F^{\prime}$ angle/ C'-F' $\cdots F$ angle and $\mathrm{F}^{\prime} \cdot \mathrm{F}^{\prime}$ distance in phase II at 0.89 and $1.0 \mathrm{GPa}$.

\begin{tabular}{llllll}
\hline & \multicolumn{2}{c}{$0.89 \mathrm{GPa}$} & \multicolumn{2}{c}{$1.0 \mathrm{GPa}$} & \multicolumn{1}{c}{ Symmetry code } \\
\hline $\mathrm{F} 2 \cdots \mathrm{F} 3$ & $124.11^{\circ} / 173.57^{\circ}$ & $2.8818(3) \AA$ & $123.79^{\circ} / 173.66^{\circ}$ & $2.8608(5) \AA$ & $1-\mathrm{x},-\mathrm{y}, 1-\mathrm{z}$ \\
$\mathrm{F} 1 \cdots \mathrm{F} 2$ & $124.46^{\circ} / 171.55^{\circ}$ & $2.9129(3) \AA$ & $124.60^{\circ} / 171.64^{\circ}$ & $2.8989(4) \AA$ & $1.5-\mathrm{x}, 0.5-\mathrm{y}, 1-\mathrm{z}$ \\
$\mathrm{F} 1 \cdots \mathrm{F} 3$ & $167.71^{\circ} / 124.26^{\circ}$ & $2.9359(4) \AA$ & $167.79^{\circ} / 124.10^{\circ}$ & $2.9270(4) \AA$ & $0.5+\mathrm{x}, 0.5+\mathrm{y}, \mathrm{z}$ \\
$\mathrm{F} 2 \cdots \mathrm{F} 3$ & $86.66^{\circ} / 81.05^{\circ}$ & $2.8818(3) \AA$ & $86.34^{\circ} / 80.67^{\circ}$ & $2.8608(5) \AA$ & $1-\mathrm{x},-\mathrm{y}, 1-\mathrm{z}$ \\
$\mathrm{F} 3 \cdots \mathrm{F} 1$ & $120.64^{\circ} / 84.91^{\circ}$ & $2.9359(4) \AA$ & $120.56^{\circ} / 84.75^{\circ}$ & $2.9270(4) \AA$ & $-0.5+\mathrm{x},-0.5+\mathrm{y}, \mathrm{z}$ \\
$\mathrm{F} 2 \cdots \mathrm{F} 1$ & $95.78^{\circ} / 79.01^{\circ}$ & $2.9129(3) \AA$ & $95.44^{\circ} / 78.59^{\circ}$ & $2.8989(4) \AA$ & $1.5-\mathrm{x}, 0.5-\mathrm{y}, 1-\mathrm{z}$ \\
\hline
\end{tabular}


Table S8. Short contacts C-F $\cdots F^{\prime}-C^{\prime}$ and their F $\cdots F$ ' distance: $C-F \cdots F$ ' angle/ $C^{\prime}-F^{\prime} \cdots F$ angle and F $\cdots F^{\prime}$ distance in phase II at 1.07 and $1.55 \mathrm{GPa}$.

\begin{tabular}{llllll}
\hline & \multicolumn{2}{c}{$1.07 \mathrm{GPa}$} & \multicolumn{2}{c}{$1.55 \mathrm{GPa}$} & \multicolumn{1}{l}{ Symmetry code } \\
\hline $\mathrm{F} 2 \cdots \mathrm{F} 3$ & $123.99^{\circ} / 173.39^{\circ}$ & $2.8491(3) \AA$ & $123.08^{\circ} / 174.57^{\circ}$ & $2.7913(3) \AA$ & $1-\mathrm{x},-\mathrm{y}, 1-\mathrm{z}$ \\
$\mathrm{F} 1 \cdots \mathrm{F} 2$ & $124.05^{\circ} / 171.36^{\circ}$ & $2.8843(3) \AA$ & $123.42^{\circ} / 172.32^{\circ}$ & $2.8195(2) \AA$ & $1.5-\mathrm{x}, 0.5-\mathrm{y}, 1-\mathrm{z}$ \\
$\mathrm{F} 1 \cdots \mathrm{F} 3$ & $167.25^{\circ} / 124.15^{\circ}$ & $2.9119(2) \AA$ & $167.94^{\circ} / 123.05^{\circ}$ & $2.8492(2) \AA$ & $0.5+\mathrm{x}, 0.5+\mathrm{y}, \mathrm{z}$ \\
$\mathrm{F} 2 \cdots \mathrm{F} 3$ & $86.39^{\circ} / 80.83^{\circ}$ & $2.8491(3) \AA$ & $85.19^{\circ} / 76.36^{\circ}$ & $2.7913(3) \AA$ & $1-\mathrm{x},-\mathrm{y}, 1-\mathrm{z}$ \\
$\mathrm{F} 3 \cdots \mathrm{F} 1$ & $120.75^{\circ} / 85.06^{\circ}$ & $2.9119(2) \AA$ & $120.56^{\circ} / 84.53^{\circ}$ & $2.8492(2) \AA$ & $-0.5+\mathrm{x},-0.5+\mathrm{y}, \mathrm{z}$ \\
$\mathrm{F} 2 \cdots \mathrm{F} 1$ & $95.64^{\circ} / 79.25^{\circ}$ & $2.8843(3) \AA$ & $94.38^{\circ} / 78.01^{\circ}$ & $2.8195(2) \AA$ & $1.5-\mathrm{x}, 0.5-\mathrm{y}, 1-\mathrm{z}$ \\
\hline
\end{tabular}

Table S9. Short contacts C-F $\cdots F^{\prime}-C^{\prime}$ and their F $\cdots F$ ' distance: C-F $\cdots F^{\prime}$ angle/ C'-F' $\cdots F$ angle and $\mathrm{F}^{\cdots} \cdot \mathrm{F}^{\prime}$ distance in phase II at 1.90 GPa.

\begin{tabular}{llll}
\hline & \multicolumn{2}{c}{$1.90 \mathrm{GPa}$} & \multicolumn{1}{l}{ Symmetry code } \\
\hline $\mathrm{F} 2 \cdots \mathrm{F} 3$ & $122.95^{\circ} / 175.22^{\circ}$ & $2.7526(2) \AA$ & $1-\mathrm{x},-\mathrm{y}, 1-\mathrm{z}$ \\
$\mathrm{F} 1 \cdots \mathrm{F} 2$ & $122.23^{\circ} / 172.23^{\circ}$ & $2.7949(2) \AA$ & $1.5-\mathrm{x}, 0.5-\mathrm{y}, 1-\mathrm{z}$ \\
$\mathrm{F} 1 \cdots \mathrm{F} 3$ & $168.48^{\circ} / 122.35^{\circ}$ & $2.8083(1) \AA$ & $0.5+\mathrm{x}, 0.5+\mathrm{y}, \mathrm{z}$ \\
$\mathrm{F} 2 \cdots \mathrm{F} 3$ & $83.98^{\circ} / 78.25^{\circ}$ & $2.7526(2) \AA$ & $1-\mathrm{x},-\mathrm{y}, 1-\mathrm{z}$ \\
$\mathrm{F} 3 \cdots \mathrm{F} 1$ & $120.17^{\circ} / 83.94^{\circ}$ & $2.8083(1) \AA$ & $-0.5+\mathrm{x},-0.5+\mathrm{y}, \mathrm{z}$ \\
$\mathrm{F} 2 \cdots \mathrm{F} 1$ & $93.35^{\circ} / 77.85^{\circ}$ & $2.7949(2) \AA$ & $1.5-\mathrm{x}, 0.5-\mathrm{y}, 1-\mathrm{z}$ \\
\hline
\end{tabular}

Table S10. Short contacts C- $X \cdots X^{\prime}-\mathrm{C}^{\prime}$ and their $X \cdots X^{\prime}$ distance: $\mathrm{C}-X^{\prime} \cdots X^{\prime}$ angle/ $\mathrm{C}^{\prime}-X^{\prime} \cdots X$ angle and $X \cdots X^{\prime}$ distance.

\begin{tabular}{|c|c|c|c|c|c|c|c|c|}
\hline & \multicolumn{2}{|r|}{ II- $\mathrm{C}_{6} \mathrm{~F}_{6}$} & \multicolumn{2}{|r|}{$\mathrm{C}_{6} \mathrm{Cl}_{6}$} & \multicolumn{2}{|r|}{$\mathrm{C}_{6} \mathrm{Br}_{6}$} & \multicolumn{2}{|r|}{$\mathrm{C}_{6} \mathrm{I}_{6}$} \\
\hline$X 2 \cdots X 3$ & $2.88 \AA$ & $173.54^{\circ} / 124.12^{\circ}$ & $3.47 \AA$ & $174.7^{\circ} / 124.17^{\circ}$ & $3.56 \AA$ & $176.97^{\circ} / 123.81^{\circ}$ & $3.94 \AA$ & $173.59^{\circ} / 123.48^{\circ}$ \\
\hline$X 1 \cdots X 2$ & $2.91 \AA$ & $171.55^{\circ} / 124.46^{\circ}$ & $3.44 \AA$ & $175.03^{\circ} / 116.73^{\circ}$ & $3.55 \AA$ & $174.12^{\circ} / 115.15^{\circ}$ & $3.70 \AA$ & $174.24^{\circ} / 114.89^{\circ}$ \\
\hline$X 3 \cdots X 1$ & $2.93 \AA$ & $167.73^{\circ} / 124.31^{\circ}$ & $3.66 \AA$ & $171.19^{\circ} / 123.27^{\circ}$ & $3.78 \AA$ & $172.99^{\circ} / 122.72^{\circ}$ & $3.71 \AA$ & $178.26^{\circ} / 124.65^{\circ} /$ \\
\hline
\end{tabular}


Table S11. Results of the PIXEL calculations for the $\mathrm{C}_{6} \mathrm{~F}_{6}$ phase II structure at $0.89 \mathrm{GPa}$.

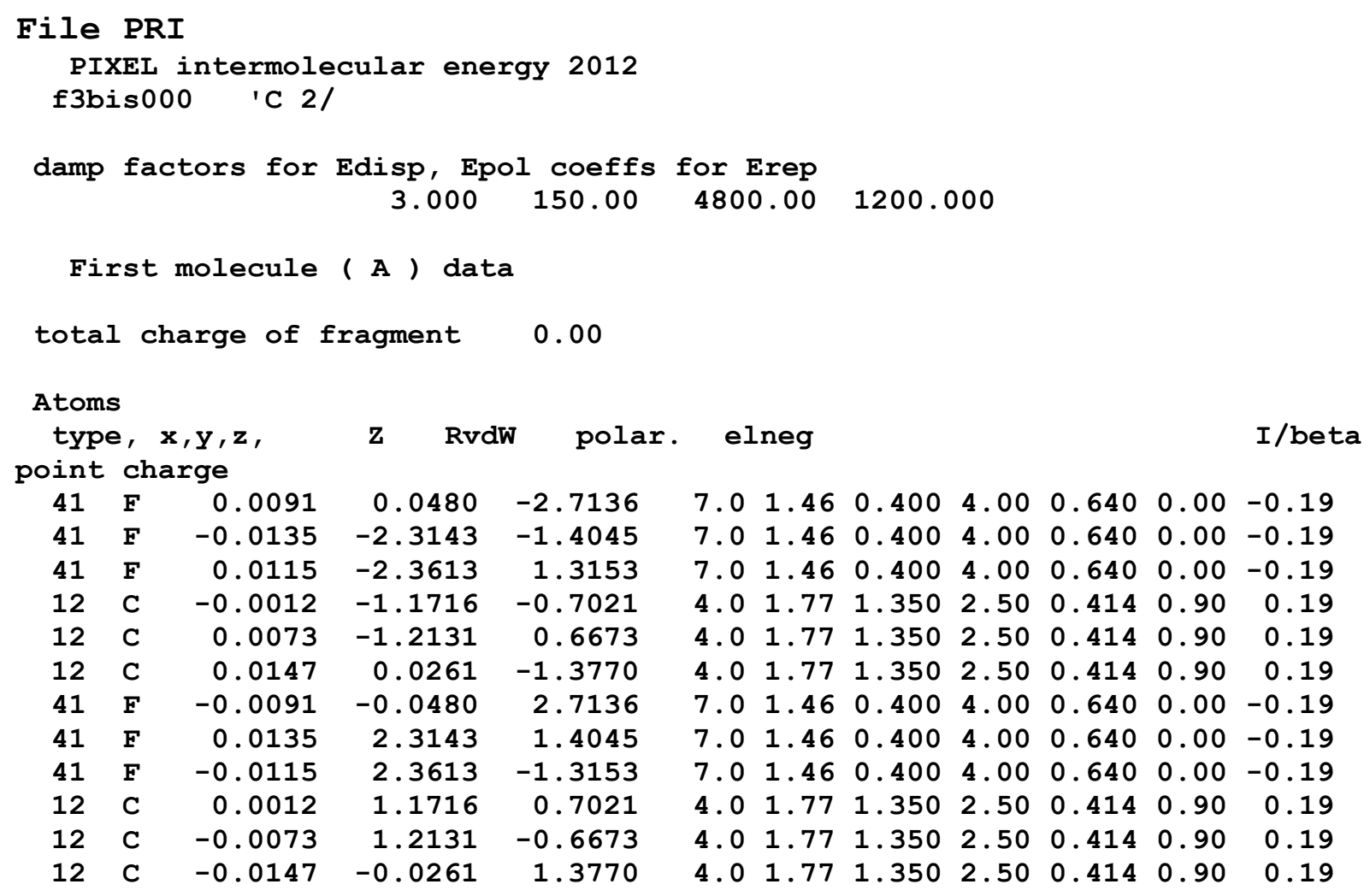

Density file title f3bis000 'C $2 /$ fragm 1

min and max original density $0.0000 \mathrm{E}+00 \quad 0.5353 \mathrm{E}+02$

$\begin{array}{lllllllll}\text { Condensation level } & 5 & & & & & & \\ \text { Density steps, original } & 100 & 140 & 140 & \text { and condensed } & 20 & 28 & 28\end{array}$

$\begin{array}{llllll}\text { steps and pixel vol (A) } & 0.4000 & 0.4000 & 0.4000 & 0.06400\end{array}$

original electron number 65.98922 remaining-pixels $65.98882 \quad 4807$

$\mathrm{q} \min$ and $\max 0.1000 \mathrm{E}-05 \quad 9999.00$

screening: electrons out low and high $0.4092 \mathrm{E}-03 \quad 0.0000 \mathrm{E}+00$

$\begin{array}{llll}\text { atom } 1 \text { condens.basin and real charge } & -7.186 & 7.000\end{array}$

atom 2 condens.basin and real charge $-6.857 \quad 7.000$

atom 3 condens.basin and real charge $-6.890 \quad 7.000$

atom 4 condens.basin and real charge $-4.306 \quad 4.000$

atom 5 condens.basin and real charge $-3.955 \quad 4.000$

atom 6 condens.basin and real charge $-3.805 \quad 4.000$

$\begin{array}{llll}\text { atom } 7 \text { condens.basin and real charge } & -7.186 & 7.000\end{array}$

atom 8 condens.basin and real charge $-6.857 \quad 7.000$

atom 9 condens.basin and real charge $-6.890 \quad 7.000$

atom 10 condens.basin and real charge $-4.306 \quad 4.000$

atom 11 condens.basin and real charge $-3.955 \quad 4.000$

atom 12 condens.basin and real charge $-3.805 \quad 4.000$

polarizability, raw, tot, renorm $0.11004 \mathrm{E}+02 \quad 0.10500 \mathrm{E}+020.10500 \mathrm{E}+02$ renormalized total charges $66.000000-66.000000$

no. of charge points per atom

$\begin{array}{lllllllllllllllll}1 & 477 & 2 & 471 & 3 & 473 & 4 & 340 & 5 & 325 & 6 & 318 & 7 & 477 & 8 & 471 & 9\end{array}$

$\begin{array}{llllll}10 & 340 & 11 & 325 & 12 & 318\end{array}$

\footnotetext{
===== Start energy calculations ===== collision parameter 0.200
} 


\begin{tabular}{|c|c|c|c|c|c|c|c|c|c|c|c|}
\hline cen & ter of & mass & dista & ance I & limi & & 0.00 & 14.00 & & & \\
\hline cell & parame & ters & $12 . \varepsilon$ & 879 & 7.38 & & .449 & 90.00 & 135.9 & 90 & 00 \\
\hline Space & group & matr & ices & & & & & & & & \\
\hline 1.0 & 0.0 & 0.0 & 0.0 & 1.0 & 0.0 & 0.0 & 0.0 & 1.0 & 0.000 & 0.000 & 0.000 \\
\hline-1.0 & 0.0 & 0.0 & 0.0 & 1.0 & 0.0 & 0.0 & 0.0 & -1.0 & 0.000 & 0.000 & 0.500 \\
\hline 1.0 & 0.0 & 0.0 & 0.0 & 1.0 & 0.0 & 0.0 & 0.0 & 1.0 & 0.500 & 0.500 & 0.000 \\
\hline-1.0 & 0.0 & 0.0 & 0.0 & 1.0 & 0.0 & 0.0 & 0.0 & -1.0 & 0.500 & 0.500 & 0.500 \\
\hline cells & $\begin{array}{l}\text { along } \\
83 \text { syr }\end{array}$ & $\begin{array}{l}a, b, c \\
m \text { ope }\end{array}$ & c for & latt & ice & arch & 7 & 7 & 7 & & \\
\hline
\end{tabular}

Computing cell dipole energy

A fragm, dipole mom.components and module, Debye

$$
\begin{array}{llll}
-0.001 & 0.001 & 0.001 & 0.002
\end{array}
$$

A fragm, dipole mom.components and module, Debye $\begin{array}{llll}0.001 & 0.001 & -0.001 & 0.002\end{array}$

A fragm, dipole mom.components and module, Debye $\begin{array}{llll}-0.001 & 0.001 & 0.001 & 0.002\end{array}$

A fragm, dipole mom.components and module, Debye $\begin{array}{llll}0.001 & 0.001 & -0.001 & 0.002\end{array}$

cell dipole moment components and module, debye

$$
\begin{array}{lllll}
-0.000 & 0.003 & -0.000 & 0.003 \text { energy } & -0.0
\end{array}
$$

A molecule, Epol, damp, no-damp $-6.0 \quad-67.9$

A...A A...B B...B total energies

$\begin{array}{lrrrr}\text { coul } & -13.0 & 0.0 & 0.0 & -13.0 \\ \text { disp } & -64.1 & 0.0 & 0.0 & -64.1 \\ \text { rep } & 40.7 & 0.0 & 0.0 & 40.7\end{array}$

$\begin{array}{rrr}40.7 & 0.0 & 0.0\end{array}$

polarization energy -6.0

Pixel coul, pol, disp, rep, tot; at-at lp, qq Ens. per mol.

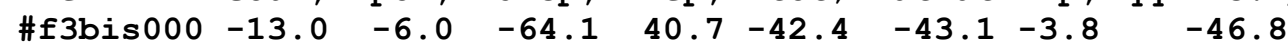

polar correction to Coulombic sums

cell dip moment(debye) and $\mathrm{E} / \mathrm{molecule} \quad 0.003 \quad-0.0$

this energy should be added to the Coulombic energy

File MLC

\begin{tabular}{|c|c|c|c|c|c|c|c|c|c|c|c|}
\hline 1.0 & 0.0 & 0.0 & 0.0 & 1.0 & 0.0 & 0.0 & 0.0 & 1.0 & 0.0000 & 0.0000 & 0.0 \\
\hline-1.0 & 0.0 & 0.0 & 0.0 & 1.0 & 0.0 & 0.0 & 0.0 & -1.0 & 0.0000 & 0.0000 & 0.5000 \\
\hline 1.0 & 0.0 & 0.0 & 0.0 & 1.0 & 0.0 & 0.0 & 0.0 & 1.0 & 0.5000 & 0.5000 & 0.000 \\
\hline-1.0 & 0.0 & 0.0 & 0.0 & .0 & 0.0 & 0.0 & 0.0 & -1.0 & 0.5000 & 0.5000 & 0 . \\
\hline 1.0 & 0.0 & 0.0 & 0.0 & .0 & 0.0 & 0.0 & 0.0 & 1.0 & -1.0000 & -1.0000 & -2.000 \\
\hline 1.0 & 0.0 & 0.0 & 0.0 & 1.0 & 0.0 & 0.0 & 0.0 & 1.0 & -1.0000 & -1.0000 & -1 \\
\hline 1.0 & 0.0 & 0.0 & 0.0 & .0 & 0.0 & 0.0 & 0.0 & 1.0 & -1.0000 & 0.0000 & -2. \\
\hline 1.0 & 0.0 & 0.0 & 0.0 & 1.0 & 0.0 & 0.0 & 0.0 & 1.0 & -1.0000 & 000 & -1 \\
\hline 1.0 & 0.0 & 0.0 & 0.0 & 1.0 & 0.0 & 0.0 & 0.0 & 1.0 & -1.0000 & 0.0000 & 0 . \\
\hline 1.0 & 0.0 & 0.0 & 0.0 & .0 & 0.0 & 0.0 & 0.0 & 1.0 & -1.0000 & 1. & -2 . \\
\hline 1.0 & 0.0 & 0.0 & 0.0 & L. 0 & 0.0 & 0.0 & 0.0 & 1.0 & -1.0000 & 1. & -1 \\
\hline 1.0 & 0.0 & 0.0 & 0.0 & 1.0 & 0.0 & 0.0 & 0.0 & 1.0 & 0.0000 & -1.0000 & -1.000 \\
\hline 1.0 & 0.0 & 0.0 & 0.0 & .0 & 0.0 & 0.0 & 0.0 & 1.0 & 0.0 & -1 & 0 . \\
\hline 1.0 & 0.0 & 0.0 & 0.0 & 1.0 & 0.0 & 0.0 & 0.0 & 1.0 & 0.0000 & -1.0000 & 1.0 \\
\hline 1.0 & 0.0 & 0.0 & 0.0 & 1.0 & 0.0 & 0.0 & 0.0 & 1.0 & 0.0000 & 0.0 & -1.000 \\
\hline 1.0 & 0.0 & 0.0 & 0.0 & .0 & 0.0 & 0.0 & 0.0 & 1.0 & 0.0000 & 0. & 1. \\
\hline 1.0 & 0.0 & 0.0 & 0.0 & 1.0 & 0.0 & 0.0 & 0.0 & 1.0 & 0.0000 & 1.0000 & -1.0000 \\
\hline 1.0 & 0.0 & 0.0 & 0.0 & 1.0 & 0.0 & 0.0 & 0.0 & 1.0 & 0.0000 & 1.0000 & 0.000 \\
\hline 1.0 & 0.0 & 0.0 & 0.0 & L. 0 & 0.0 & 0.0 & 0.0 & 1.0 & 0.0000 & 1.0000 & 1. \\
\hline 1.0 & 0.0 & 0.0 & 0.0 & 1.0 & 0.0 & 0.0 & 0.0 & 1.0 & 1.0000 & -1.0000 & 1.000 \\
\hline 1.0 & 0.0 & 0.0 & 0.0 & 1.0 & 0.0 & 0.0 & 0.0 & 1.0 & 1.0000 & -1.0000 & 2.000 \\
\hline 1.0 & 0.0 & 0.0 & 0.0 & L. 0 & 0.0 & 0.0 & 0.0 & 1.0 & 1.0000 & 0.0 & 0. \\
\hline 1.0 & 0.0 & 0.0 & 0.0 & 1.0 & 0.0 & 0.0 & 0.0 & 1.0 & 1.0000 & 0.0000 & 1.000 \\
\hline 1.0 & 0.0 & 0.0 & 0.0 & 1.0 & 0.0 & 0.0 & 0.0 & 1.0 & 1.0000 & 0.0000 & 2.000 \\
\hline
\end{tabular}

183 f3bis000 'C 2/ symmetry operations 


\begin{tabular}{|c|c|c|c|c|c|c|c|c|c|c|c|c|}
\hline 25 & 1.0 & .0 & 0.0 & 0.0 & 1.0 & 0.0 & 0.0 & 0.0 & 1.0 & 000 & 000 & 000 \\
\hline 26 & 1.0 & .0 & 0.0 & 0.0 & 1.0 & 0.0 & 0.0 & 0.0 & 1.0 & 1.0000 & 1.0000 & 2.0000 \\
\hline בר & -1.0 & .0 & .0 & 0.0 & 1.0 & .0 & 0.0 & 0.0 & -1.0 & .0000 & -1.0000 & 0 \\
\hline 28 & -1.0 & .0 & 0.0 & 0.0 & 1.0 & 0.0 & 0.0 & 0.0 & -1.0 & 0.0000 & -1.0000 & 0.5000 \\
\hline 29 & -1.0 & 0.0 & 0.0 & 0.0 & 1.0 & 0.0 & 0.0 & 0.0 & -1.0 & 0.0000 & 0.0000 & -0.5000 \\
\hline 30 & -1.0 & .0 & 0.0 & 0.0 & 1.0 & 0.0 & 0.0 & 0.0 & -1.0 & & 000 & \\
\hline 31 & -1.0 & .0 & 0.0 & 0.0 & 1.0 & 0.0 & 0.0 & 0.0 & -1.0 & 0.0000 & 1.0000 & 0.5000 \\
\hline 32 & -1.0 & 0.0 & 0.0 & 0.0 & 1.0 & 0.0 & 0.0 & 0.0 & -1.0 & 1.0000 & -1.0000 & 0.5000 \\
\hline 33 & -1.0 & 0 & 0.0 & 0.0 & 1.0 & 0.0 & 0.0 & 0.0 & -1.0 & & 000 & 000 \\
\hline 34 & -1.0 & .0 & 0.0 & 0.0 & 1.0 & 0.0 & 0.0 & 0.0 & -1.0 & 1.0000 & 0.0000 & -0.5000 \\
\hline 35 & -1.0 & 0.0 & 0.0 & 0.0 & 1.0 & 0.0 & 0.0 & 0.0 & -1.0 & 1.0000 & 0.0000 & 0.5000 \\
\hline 36 & -1.0 & .0 & 0.0 & 0.0 & 1.0 & 0.0 & 0.0 & 0.0 & -1.0 & 1.0000 & 0.0000 & 1.5000 \\
\hline 37 & -1.0 & .0 & 0.0 & 0.0 & 1.0 & 0.0 & 0.0 & 0.0 & -1.0 & & & \\
\hline 38 & -1.0 & 0.0 & 0.0 & 0.0 & 1.0 & 0.0 & 0.0 & 0.0 & -1.0 & 1.0000 & 1.0000 & 0.5000 \\
\hline 39 & -1.0 & .0 & 0.0 & 0.0 & 1.0 & 0.0 & 0.0 & 0.0 & -1.0 & 1.0000 & 1.0000 & 1.5000 \\
\hline 40 & -1.0 & .0 & 0.0 & 0.0 & 1.0 & .0 & 0.0 & 0.0 & -1.0 & & -1 & \\
\hline 41 & -1.0 & .0 & 0.0 & 0.0 & 1.0 & 0.0 & 0.0 & 0.0 & -1.0 & 2.0000 & -1.0000 & 2.5000 \\
\hline 42 & -1.0 & 0.0 & 0.0 & 0.0 & 1.0 & 0.0 & 0.0 & 0.0 & -1.0 & 2.0000 & 0.0000 & 1.5000 \\
\hline 43 & -1.0 & .0 & 0.0 & 0.0 & 1.0 & 0.0 & 0.0 & 0.0 & -1.0 & 00 & 0.0000 & 000 \\
\hline 44 & -1.0 & 0.0 & 0.0 & 0.0 & 1.0 & 0.0 & 0.0 & 0.0 & -1.0 & 00 & 000 & \\
\hline 45 & -1.0 & .0 & 0.0 & 0.0 & 1.0 & 0.0 & 0.0 & 0.0 & -1.0 & 2.0000 & 1.0000 & 000 \\
\hline 46 & 1.0 & .0 & 0.0 & 0.0 & 1.0 & 0.0 & 0.0 & 0.0 & 1.0 & -0 . & -1.5000 & -1.0000 \\
\hline 47 & 1.0 & 0.0 & 0.0 & 0.0 & 1.0 & 0.0 & 0.0 & 0.0 & 1.0 & -0 . & -1 & \\
\hline 48 & 1.0 & 0.0 & 0.0 & 0.0 & 1.0 & 0.0 & 0.0 & 0.0 & 1.0 & -0 . & -0 . & -2.0000 \\
\hline 49 & 1.0 & 0.0 & 0.0 & 0. & 1.0 & 0.0 & 0.0 & 0.0 & 1.0 & -0 & -0 . & -1 \\
\hline 50 & 1.0 & 0.0 & 0.0 & 0.0 & 1.0 & 0.0 & 0.0 & 0.0 & 1.0 & -0 . & -0.5000 & 0.0000 \\
\hline 51 & 1.0 & 0.0 & 0.0 & 0.0 & 10 & 0.0 & 0.0 & 0.0 & 1.0 & -0 . & 00 & -2.0000 \\
\hline 52 & 1.0 & 0.0 & 0.0 & 0.0 & 1.0 & 0.0 & 0.0 & 0.0 & 1.0 & -0 & 00 & -1 \\
\hline 53 & 1.0 & 0.0 & 0.0 & 0. & 1.0 & 0.0 & 0.0 & 0.0 & 1.0 & -0 . & 00 & 00 \\
\hline 54 & 1.0 & 0.0 & 0.0 & 0.0 & 1.0 & 0.0 & 0.0 & 0.0 & 1.0 & -0 & 000 & -1 \\
\hline 55 & 1.0 & 0.0 & 0.0 & 0.0 & 1.0 & 0.0 & 0.0 & 0.0 & 1.0 & -0 . & 00 & 00 \\
\hline 56 & 1.0 & .0 & 0.0 & 0. & 1.0 & 0.0 & 0.0 & 0.0 & 1.0 & 0.5000 & -1 & 00 \\
\hline 57 & 1.0 & 0.0 & 0. & 0 . & 1.0 & 0.0 & 0.0 & 0.0 & 1.0 & 0. & -1 & 00 \\
\hline 58 & 1.0 & 0. & 0 . & 0 & 1.0 & 0.0 & 0.0 & 0. & 1.0 & & -0 . & \\
\hline 59 & 1.0 & .0 & 0. & 0. & 1.0 & .0 & 0.0 & 0.0 & 1.0 & 00 & -0 . & 00 \\
\hline 60 & 1.0 & $0 . c$ & 0. & 0. & 1.0 & 0.0 & 0.0 & 0.0 & 1.0 & 0. & -0.5000 & 00 \\
\hline 61 & 1.0 & 0 . & & & 1. & 0.0 & 0.0 & 0 & 1.0 & & & \\
\hline 62 & 1.0 & 0.0 & 0 & 0 & 1.0 & 0 & 0.0 & 0.0 & 1.0 & 0 & 00 & 2. \\
\hline 63 & 1.0 & $0 . c$ & 0. & 0. & 1.0 & 0.0 & 0.0 & 0.0 & 1.0 & 00 & 000 & 00 \\
\hline 64 & 1.0 & 0.0 & & & 1. & 0.0 & 0.0 & & 1.0 & & & \\
\hline 65 & -1.0 & 0.0 & 0 & 0. & 1. & .0 & 0.0 & 0.0 & -1.0 & -0 & -0 . & -0 \\
\hline 66 & -1.0 & 0. & 0. & 0 & 1.0 & 0.0 & 0.0 & 0.0 & -1.0 & -0 & 00 & -0 \\
\hline 67 & -1.0 & .0 & 0. & 0 & 1.0 & 0.0 & 0.0 & 0.0 & -1.0 & & -1 & \\
\hline 68 & -1.0 & & & & 1.0 & 0.0 & 0.0 & 0.0 & -1.0 & & -0 . & -0 . \\
\hline 69 & -1.0 & 0.0 & 0 . & 0 & 1.0 & .0 & 0.0 & 0.0 & -1.0 & 0 & -0 . & 00 \\
\hline 70 & -1.0 & 0.0 & 0.0 & 0.0 & 1.0 & 0.0 & 0.0 & 0.0 & -1.0 & & -0 . & \\
\hline 71 & -1.0 & 0.0 & & & 1. & 0.0 & 0.0 & 0.0 & -1.0 & & & -0 . \\
\hline 72 & -1.0 & 0 . & 0 & 0 & 1.0 & 0 & 0.0 & 0.0 & -1.0 & 0 & 00 & 00 \\
\hline 73 & -1.0 & 0.0 & 0.0 & 0.0 & 1.0 & 0.0 & 0.0 & 0.0 & -1.0 & 00 & 00 & 0.5000 \\
\hline 74 & -1.0 & 0 & 0.0 & 0 & 1. & 0.0 & 0.0 & 0.0 & -1.0 & & & \\
\hline 75 & -1.0 & 0. & 0 . & 0 & 1. & 0 & 0.0 & 0.0 & -1.0 & 0 & -0 . & 00 \\
\hline 76 & -1.0 & 0.0 & 0.0 & 0 . & 1.0 & 0.0 & 0.0 & 0.0 & -1.0 & 1.5000 & -0.5000 & 1.5000 \\
\hline 77 & -1.0 & 0.0 & 0. & & 1. & & 0.0 & 0.0 & -1.0 & & -0 . & \\
\hline 78 & -1.0 & 0. & 0 . & 0 & 1. & 0.0 & 0.0 & 0.0 & -1.0 & & & \\
\hline 79 & -1.0 & 0.0 & 0.0 & 0. & 1.0 & 0.0 & 0.0 & 0.0 & -1.0 & 1.5000 & 0.5000 & 1.5000 \\
\hline 80 & -1.0 & $0 . c$ & 0.0 & 0. & 1.0 & 0.0 & 0.0 & 0.0 & -1.0 & 00 & 00 & \\
\hline 81 & -1.0 & .0 & 0.0 & 0. & 1.0 & 0.0 & 0.0 & 0.0 & -1.0 & & 00 & \\
\hline 82 & -1.0 & 0.0 & 0.0 & 0. & 1.0 & 0. & 0.0 & 0.0 & -1.0 & 2.5000 & -0.5000 & 2.5000 \\
\hline 83 & -1. & 0.0 & .0 & 0.0 & 1.0 & 0.0 & 0.0 & 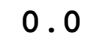 & -1.0 & 2.5000 & 0.5000 & 2.300 \\
\hline & & -6.0 & & & 10 & & & A. & & -3.8 & -46.8 & \\
\hline
\end{tabular}




\begin{tabular}{|c|c|c|c|c|c|c|c|c|c|}
\hline 1 & 2 & 10.272 & 0.0 & -0.0 & -0.2 & 0.0 & -0.1 & -0.4 & 0.2 \\
\hline 1 & 3 & 7.423 & 0.2 & -0.0 & -4.6 & 1.6 & -2.9 & -1.2 & 1.9 \\
\hline & 4 & 5.817 & -4.4 & -1.1 & -11.4 & 7.3 & -9.7 & -5.8 & -6.3 \\
\hline & 5 & 13.897 & -0.0 & -0.0 & -0.0 & 0.0 & -0.0 & -0.0 & -0.0 \\
\hline & 6 & 11.635 & -0.0 & -0.0 & -0.0 & 0.0 & -0.1 & -0.1 & -0.1 \\
\hline & 7 & 11.774 & 0.1 & -0.0 & -0.0 & 0.0 & 0.0 & -0.1 & 0.3 \\
\hline 1 & 8 & 8.992 & -0.2 & -0.0 & -0.2 & 0.0 & -0.4 & -0.6 & -0.6 \\
\hline 1 & 9 & 12.879 & 0.0 & -0.0 & -0.0 & 0.0 & -0.0 & -0.1 & 0.1 \\
\hline 1 & 10 & 13.897 & 0.0 & -0.0 & -0.0 & 0.0 & -0.0 & -0.0 & 0.0 \\
\hline 1 & 11 & 11.635 & -0.0 & -0.0 & -0.0 & 0.0 & -0.1 & -0.1 & -0.1 \\
\hline 1 & 12 & 11.220 & -0.1 & -0.0 & -0.1 & 0.0 & -0.1 & -0.2 & -0.2 \\
\hline 1 & 13 & 7.383 & 0.2 & -0.1 & -4.8 & 2.1 & -2.5 & -0.5 & 2.1 \\
\hline 1 & 14 & 11.220 & -0.0 & -0.0 & -0.1 & 0.0 & -0.1 & -0.2 & -0.2 \\
\hline 1 & 15 & 8.449 & -0.1 & -0.0 & -0.3 & 0.0 & -0.4 & -0.7 & -0.4 \\
\hline 1 & 16 & 8.449 & -0.1 & -0.0 & -0.3 & 0.0 & -0.4 & -0.7 & -0.4 \\
\hline 1 & 17 & 11.220 & -0.0 & -0.0 & -0.1 & 0.0 & -0.1 & -0.2 & -0.2 \\
\hline 1 & 18 & 7.383 & 0.2 & -0.1 & -4.8 & 2.1 & -2.5 & -0.5 & 2.1 \\
\hline 1 & 19 & 11.220 & -0.1 & -0.0 & -0.1 & 0.0 & -0.1 & -0.2 & -0.2 \\
\hline 1 & 20 & 11.635 & -0.0 & -0.0 & -0.0 & 0.0 & -0.1 & -0.1 & -0.1 \\
\hline 1 & 21 & 13.897 & 0.0 & -0.0 & -0.0 & 0.0 & -0.0 & -0.0 & 0.0 \\
\hline 1 & 22 & 12.879 & 0.0 & -0.0 & -0.0 & 0.0 & -0.0 & -0.1 & 0.1 \\
\hline 1 & 23 & 8.992 & -0.2 & -0.0 & -0.2 & 0.0 & -0.4 & -0.6 & -0.6 \\
\hline 1 & 24 & 11.774 & 0.1 & -0.0 & -0.0 & 0.0 & 0.0 & -0.1 & 0.3 \\
\hline 1 & 25 & 11.635 & -0.0 & -0.0 & -0.0 & 0.0 & -0.1 & -0.1 & -0.1 \\
\hline 1 & 26 & 13.897 & -0.0 & -0.0 & -0.0 & 0.0 & -0.0 & -0.0 & -0.0 \\
\hline 1 & 27 & 12.100 & -0.0 & -0.0 & -0.0 & 0.0 & -0.1 & -0.1 & -0.1 \\
\hline 1 & 28 & 12.650 & 0.0 & -0.0 & -0.0 & 0.0 & -0.0 & -0.1 & 0.1 \\
\hline 1 & 29 & 9.586 & 0.1 & -0.0 & -0.1 & 0.0 & -0.0 & -0.3 & 0.2 \\
\hline 1 & 30 & 12.100 & -0.0 & -0.0 & -0.0 & 0.0 & -0.1 & -0.1 & -0.1 \\
\hline 1 & 31 & 12.650 & 0.0 & -0.0 & -0.0 & 0.0 & -0.0 & -0.1 & 0.1 \\
\hline 1 & 32 & 8.506 & -0.1 & -0.0 & -0.6 & 0.0 & -0.7 & -1.2 & -0.4 \\
\hline 1 & 33 & 8.506 & -0.1 & -0.0 & -0.6 & 0.0 & -0.7 & -1.2 & -0.4 \\
\hline 1 & 34 & 12.674 & -0.0 & -0.0 & -0.0 & 0.0 & -0.0 & -0.1 & -0.1 \\
\hline 1 & 35 & 4.225 & -4.9 & -4.4 & -22.8 & 20.4 & -11.7 & -18.1 & 3.2 \\
\hline 1 & 36 & 4.224 & -4.9 & -4.4 & -22.8 & 20.6 & -11.5 & -18.1 & 3.2 \\
\hline 1 & 37 & 12.673 & -0.0 & -0.0 & -0.0 & 0.0 & -0.0 & -0.1 & -0.1 \\
\hline 1 & 38 & 8.506 & -0.1 & -0.0 & -0.6 & 0.0 & -0.7 & -1.2 & -0.4 \\
\hline 1 & 39 & 8.506 & -0.1 & -0.0 & -0.6 & 0.0 & -0.7 & -1.2 & -0.4 \\
\hline 1 & 40 & 12.650 & 0.0 & -0.0 & -0.0 & 0.0 & -0.0 & -0.1 & 0.1 \\
\hline 1 & 41 & 12.100 & -0.0 & -0.0 & -0.0 & 0.0 & -0.1 & -0.1 & -0.1 \\
\hline 1 & 42 & 10.272 & 0.0 & -0.0 & -0.2 & 0.0 & -0.1 & -0.4 & 0.2 \\
\hline 1 & 43 & 9.586 & 0.1 & -0.0 & -0.1 & 0.0 & -0.0 & -0.3 & 0.2 \\
\hline 1 & 44 & 12.650 & 0.0 & -0.0 & -0.0 & 0.0 & -0.0 & -0.1 & 0.1 \\
\hline 1 & 45 & 12.100 & -0.0 & -0.0 & -0.0 & 0.0 & -0.1 & -0.1 & -0.1 \\
\hline 1 & 46 & 12.542 & -0.0 & -0.0 & -0.0 & 0.0 & -0.0 & -0.1 & -0.1 \\
\hline 1 & 47 & 12.811 & 0.0 & -0.0 & -0.0 & 0.0 & -0.0 & -0.1 & 0.1 \\
\hline 1 & 48 & 13.574 & 0.0 & -0.0 & -0.0 & 0.0 & -0.0 & -0.0 & 0.0 \\
\hline 1 & 49 & 6.949 & 0.2 & -0.0 & -0.7 & 0.0 & -0.6 & -1.6 & 0.4 \\
\hline 1 & 50 & 7.423 & 0.2 & -0.0 & -4.6 & 1.6 & -2.9 & -1.2 & 1.9 \\
\hline 1 & 51 & 13.574 & 0.0 & -0.0 & -0.0 & 0.0 & 0.0 & -0.0 & 0.0 \\
\hline 1 & 52 & 6.949 & 0.2 & -0.0 & -0.7 & 0.0 & -0.5 & -1.6 & 0.7 \\
\hline 1 & 53 & 7.423 & 0.4 & -0.1 & -4.6 & 1.8 & -2.5 & -0.8 & 2.2 \\
\hline 1 & 54 & 12.542 & -0.0 & -0.0 & -0.0 & 0.0 & -0.1 & -0.1 & -0.1 \\
\hline 1 & 55 & 12.811 & 0.0 & -0.0 & -0.0 & 0.0 & 0.0 & -0.1 & 0.1 \\
\hline 1 & 56 & 12.811 & 0.0 & -0.0 & -0.0 & 0.0 & 0.0 & -0.1 & 0.1 \\
\hline 1 & 57 & 12.542 & -0.0 & -0.0 & -0.0 & 0.0 & -0.1 & -0.1 & -0.1 \\
\hline 1 & 58 & 7.423 & 0.4 & -0.1 & -4.6 & 1.8 & -2.5 & -0.8 & 2.2 \\
\hline 1 & 59 & 6.949 & 0.2 & -0.0 & -0.7 & 0.0 & -0.5 & -1.6 & 0.7 \\
\hline 1 & 60 & 13.574 & 0.0 & -0.0 & -0.0 & 0.0 & 0.0 & -0.0 & 0.0 \\
\hline 1 & 61 & 6.949 & 0.2 & -0.0 & -0.7 & 0.0 & -0.6 & -1.6 & 0.4 \\
\hline 1 & 62 & 13.574 & 0.0 & -0.0 & -0.0 & 0.0 & -0.0 & -0.0 & 0.0 \\
\hline
\end{tabular}




$\begin{array}{rrrrrrrrrr}1 & 63 & 12.811 & 0.0 & -0.0 & -0.0 & 0.0 & -0.0 & -0.1 & 0.1 \\ 1 & 64 & 12.542 & -0.0 & -0.0 & -0.0 & 0.0 & -0.0 & -0.1 & -0.1 \\ 1 & 65 & 13.985 & -0.0 & -0.0 & -0.0 & 0.0 & -0.0 & -0.1 & -0.1 \\ 1 & 66 & 13.985 & -0.0 & -0.0 & -0.0 & 0.0 & -0.0 & -0.1 & -0.1 \\ 1 & 67 & 11.952 & 0.0 & -0.0 & -0.0 & 0.0 & -0.0 & -0.1 & 0.1 \\ 1 & 68 & 9.921 & 0.1 & -0.0 & -0.1 & 0.0 & -0.0 & -0.2 & 0.2 \\ 1 & 69 & 5.817 & -4.4 & -1.1 & -11.4 & 7.3 & -9.7 & -5.8 & -6.3 \\ 1 & 70 & 10.585 & -0.0 & -0.0 & -0.1 & 0.0 & -0.1 & -0.3 & -0.1 \\ 1 & 71 & 9.921 & 0.1 & -0.0 & -0.1 & 0.0 & -0.0 & -0.2 & 0.2 \\ 1 & 72 & 10.585 & -0.0 & -0.0 & -0.1 & 0.0 & -0.1 & -0.3 & -0.1 \\ 1 & 73 & 11.952 & 0.0 & -0.0 & -0.0 & 0.0 & -0.0 & -0.1 & 0.1 \\ 1 & 74 & 11.952 & 0.0 & -0.0 & -0.0 & 0.0 & -0.0 & -0.1 & 0.1 \\ 1 & 75 & 10.585 & -0.0 & -0.0 & -0.1 & 0.0 & -0.1 & -0.3 & -0.1 \\ 1 & 76 & 5.817 & -4.4 & -1.1 & -11.4 & 7.5 & -9.5 & -5.8 & -6.3 \\ 1 & 77 & 9.921 & 0.1 & -0.0 & -0.1 & 0.0 & -0.0 & -0.2 & 0.2 \\ 1 & 78 & 10.585 & -0.0 & -0.0 & -0.1 & 0.0 & -0.1 & -0.3 & -0.1 \\ 1 & 79 & 5.817 & -4.4 & -1.1 & -11.4 & 7.5 & -9.5 & -5.8 & -6.3 \\ 1 & 80 & 9.921 & 0.1 & -0.0 & -0.1 & 0.0 & -0.0 & -0.2 & 0.2 \\ 1 & 81 & 11.952 & 0.0 & -0.0 & -0.0 & 0.0 & -0.0 & -0.1 & 0.1 \\ 1 & 82 & 13.985 & -0.0 & -0.0 & -0.0 & 0.0 & -0.0 & -0.1 & -0.1 \\ 1 & 83 & 13.985 & -0.0 & -0.0 & -0.0 & 0.0 & -0.0 & -0.1 & -0.1\end{array}$

Table S12. Results of the PIXEL calculations for the C6F6 phase II structure at $1.90 \mathrm{GPa}$.

File PRI

PIXEL intermolecular energy 2012

f19GPaxx 'C $2 / c$ '

damp factors for Edisp, Epol coeffs for Erep

$\begin{array}{llll}3.000 & 150.00 \quad 4800.00 & 1200.000\end{array}$

First molecule ( A ) data

total charge of fragment 0.00

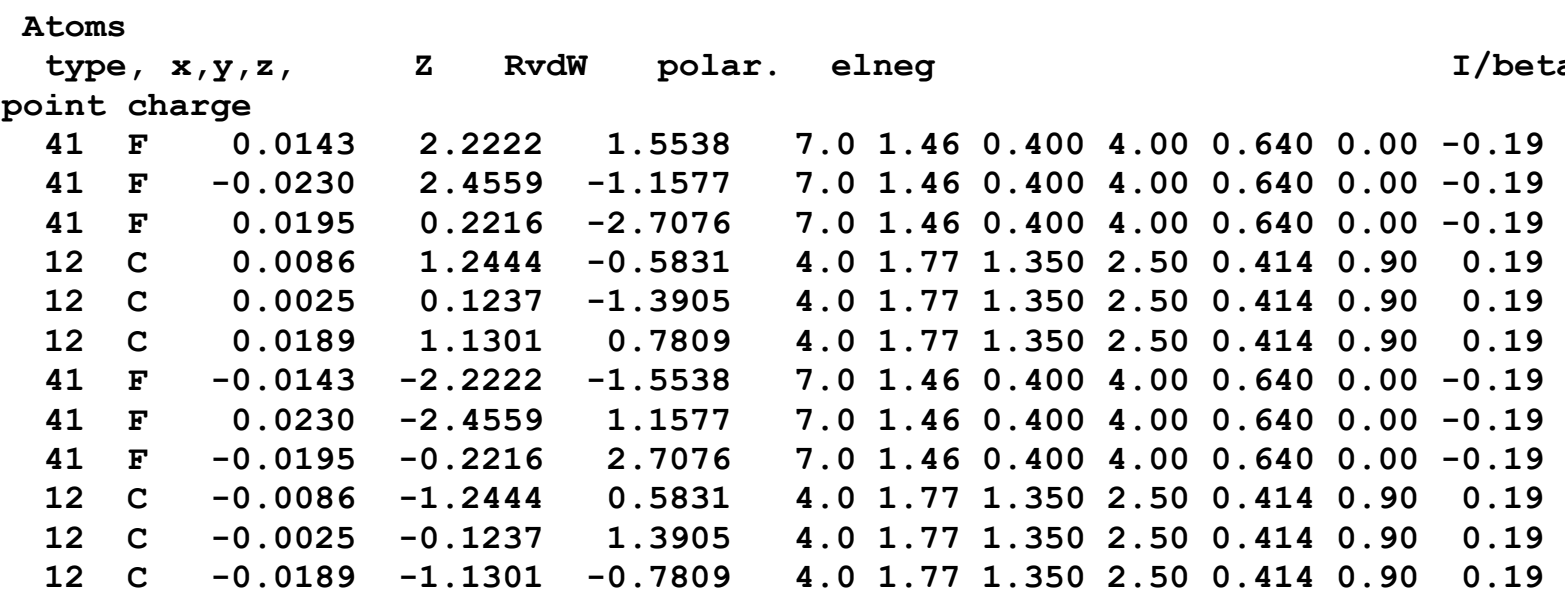

Density file title f19GPaxx 'C 2/c' fragm 1

min and max original density $0.0000 \mathrm{E}+00 \quad 0.6591 \mathrm{E}+02$

Condensation level 5

$\begin{array}{llllllll}\text { Density steps, original } & 100 & 140 & 140 & \text { and condensed } & 20 & 28 & 28\end{array}$

$\begin{array}{llllll}\text { steps and pixel vol (A) } \quad 0.4000 & 0.4000 & 0.4000 & 0.06400\end{array}$

original electron number 66.00741 remaining-pixels $66.00702 \quad 4836$ 


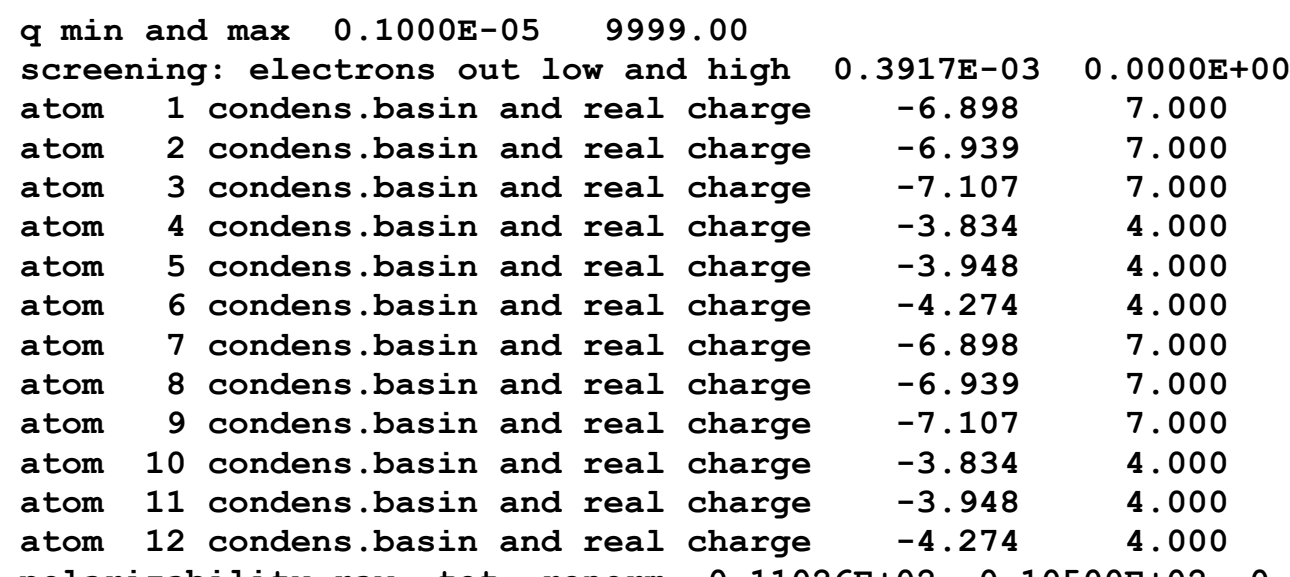

polarizability, raw, tot, renorm $0.11026 \mathrm{E}+020.10500 \mathrm{E}+020.10500 \mathrm{E}+02$ renormalized total charges $66.000000-66.000000$

no. of charge points per atom

$\begin{array}{llllllllllllllllll}1 & 472 & 2 & 490 & 3 & 467 & 4 & 323 & 5 & 333 & 6 & 333 & 7 & 472 & 8 & 490 & 9 & 467\end{array}$

$\begin{array}{llllll}10 & 323 & 11 & 333 & 12 & 333\end{array}$

\begin{tabular}{|c|c|c|c|c|c|c|c|c|c|c|c|}
\hline \multicolumn{12}{|c|}{$\begin{aligned}= & ===\text { Start energy ca } \\
& \text { collision parameter }\end{aligned}$} \\
\hline \multicolumn{2}{|c|}{ center } & mass & \multicolumn{2}{|c|}{ distance } & \multicolumn{2}{|c|}{ limits } & 0.00 & 14.00 & & & \\
\hline cell & parame & eters & \multicolumn{2}{|c|}{12.630} & \multicolumn{2}{|c|}{7.260} & 8.146 & 90.00 & 136.34 & \multicolumn{2}{|c|}{90.00} \\
\hline \multicolumn{12}{|c|}{ Space group matrices } \\
\hline 1.0 & 0.0 & 0.0 & 0.0 & 1.0 & 0.0 & 0.0 & 0.0 & 1.0 & 0.000 & 0.000 & 0.000 \\
\hline-1.0 & 0.0 & 0.0 & 0.0 & 1.0 & 0.0 & 0.0 & 0.0 & -1.0 & 0.000 & 0.000 & 0.500 \\
\hline 1.0 & 0.0 & 0.0 & 0.0 & 1.0 & 0.0 & 0.0 & 0.0 & 1.0 & 0.500 & 0.500 & 0.000 \\
\hline-1.0 & 0.0 & 0.0 & 0.0 & 1.0 & 0.0 & 0.0 & 0.0 & -1.0 & 0.500 & 0.500 & 0.500 \\
\hline cells & along & $a, b, c$ & $c$ for & latt & tice s & search & 7 & 7 & 7 & & \\
\hline
\end{tabular}

Computing cell dipole energy

A fragm, dipole mom.components and module, Debye

$$
\begin{array}{llll}
0.001 & 0.000 & 0.001 & 0.002
\end{array}
$$

A fragm, dipole mom.components and module, Debye $\begin{array}{llll}-0.001 & 0.000 & -0.001 & 0.002\end{array}$

A fragm, dipole mom.components and module, Debye $\begin{array}{llll}0.001 & 0.000 & 0.001 & 0.002\end{array}$

A fragm, dipole mom.components and module, Debye $\begin{array}{llll}-0.001 & 0.000 & -0.001 & 0.002\end{array}$

cell dipole moment components and module, debye
$-0.000$
$0.002-0.000$
0.002 energy
$-0.0$

A molecule, Epol, damp, no-damp $-10.0 \quad-136.5$

$\begin{array}{lrrrr}\text { A...A } & \text { A...B } & \text { B. . B total energies } \\ \text { coul } & -24.1 & 0.0 & 0.0 & -24.1 \\ \text { disp } & -80.3 & 0.0 & 0.0 & -80.3 \\ \text { rep } & 78.3 & 0.0 & 0.0 & 78.3\end{array}$

polarization energy -10.0

Pixel coul pol disp rep tot at-at lp qq Ens. per mol. $\begin{array}{lllllllll}\text { \#f19GPaxx } & -24.1 & -10.0 & -80.3 & 78.3 & -36.1 & -32.6 & -4.2 & -36.8\end{array}$

polar correction to Coulombic sums

cell dip moment(debye) and $\mathrm{E} / \mathrm{molecule} \quad 0.002 \quad-0.0$

this energy should be added to the Coulombic energy

File MLC

191 f19GPaxx 'C $2 / c$ ' symmetry operations 


\begin{tabular}{|c|c|c|c|c|c|c|c|c|c|c|c|c|}
\hline 1 & 1.0 & 0.0 & 0.0 & 0.0 & 1.0 & 0.0 & 0.0 & 0.0 & 1.0 & 0.0000 & 0.0000 & 0.0000 \\
\hline 2 & -1.0 & 0.0 & 0.0 & 0.0 & 1.0 & 0.0 & 0.0 & 0.0 & -1.0 & 0.0000 & 0.0000 & 0.5000 \\
\hline 3 & 1.0 & 0.0 & 0.0 & 0.0 & 1.0 & 0.0 & 0.0 & 0.0 & 1.0 & 0.5000 & 0.5000 & 0.0000 \\
\hline 4 & -1.0 & 0.0 & 0.0 & 0.0 & 1.0 & 0.0 & 0.0 & 0.0 & -1.0 & 0.5000 & 0.5000 & 0.5000 \\
\hline 5 & 1.0 & 0.0 & 0.0 & 0.0 & 1.0 & 0.0 & 0.0 & 0.0 & 1.0 & -1.0000 & -1.0000 & -2.0000 \\
\hline 6 & 1.0 & 0.0 & 0.0 & 0.0 & 1.0 & 0.0 & 0.0 & 0.0 & 1.0 & -1.0000 & -1.0000 & -1.0000 \\
\hline 7 & 1.0 & 0.0 & 0.0 & 0.0 & 1.0 & 0.0 & 0.0 & 0.0 & 1.0 & -1.0000 & 0.0000 & -2.0000 \\
\hline 8 & 1.0 & 0.0 & 0.0 & 0.0 & 1.0 & 0.0 & 0.0 & 0.0 & 1.0 & -1.0000 & 0.0000 & -1.0000 \\
\hline 9 & 1.0 & 0.0 & 0.0 & 0.0 & 1.0 & 0.0 & 0.0 & 0.0 & 1.0 & -1.0000 & 0.0000 & 0.0000 \\
\hline 10 & 1.0 & 0.0 & 0.0 & 0.0 & 1.0 & 0.0 & 0.0 & 0.0 & 1.0 & -1 & 000 & -2.0000 \\
\hline 11 & 1.0 & 0.0 & 0.0 & 0.0 & 1.0 & 0.0 & 0.0 & 0.0 & 1.0 & -1.0000 & 1.0000 & -1.0000 \\
\hline 12 & 1.0 & 0.0 & 0.0 & 0.0 & 1.0 & 0.0 & 0.0 & 0.0 & 1.0 & 0.0000 & -1.0000 & -1.0000 \\
\hline 13 & 1.0 & 0.0 & 0.0 & 0.0 & 1.0 & 0.0 & 0.0 & 0.0 & 1.0 & 0.0000 & -1.0000 & 0.0000 \\
\hline 14 & 1.0 & 0.0 & 0.0 & 0.0 & 1.0 & 0.0 & 0.0 & 0.0 & 1.0 & 0.0000 & -1.0000 & 1.0000 \\
\hline 15 & 1.0 & 0.0 & 0.0 & 0.0 & 1.0 & 0.0 & 0.0 & 0.0 & 1.0 & 0.0000 & 0.0000 & -1.0000 \\
\hline 16 & 1.0 & 0.0 & 0.0 & 0.0 & 1.0 & 0.0 & 0.0 & 0.0 & 1.0 & 0.0000 & 0.0000 & 1.0000 \\
\hline 17 & 1.0 & 0.0 & 0.0 & 0.0 & 1.0 & 0.0 & 0.0 & 0.0 & 1.0 & 0.0000 & 1.0000 & -1.0000 \\
\hline 18 & 1.0 & 0.0 & 0.0 & 0.0 & 1.0 & 0.0 & 0.0 & 0.0 & 1.0 & 0.0000 & 1.0000 & 0.0000 \\
\hline 19 & 1.0 & 0.0 & 0.0 & 0.0 & 1.0 & 0.0 & 0.0 & 0.0 & 1.0 & 0.0000 & 1.0000 & 1.0000 \\
\hline 20 & 1.0 & 0.0 & 0.0 & 0.0 & 1.0 & 0.0 & 0.0 & 0.0 & 1.0 & 1.0000 & -1.0000 & 1.0000 \\
\hline 21 & 1.0 & 0.0 & 0.0 & 0.0 & 1.0 & 0.0 & 0.0 & 0.0 & 1.0 & 1.0000 & -1.0000 & 2.0000 \\
\hline 22 & 1.0 & 0.0 & 0.0 & 0.0 & 1.0 & 0.0 & 0.0 & 0.0 & 1.0 & 1.0000 & 0.0000 & 0.0000 \\
\hline 23 & 1.0 & 0.0 & 0.0 & 0.0 & 1.0 & 0.0 & 0.0 & 0.0 & 1.0 & 1.0000 & 0.0000 & 1.0000 \\
\hline 24 & 1.0 & 0.0 & 0.0 & 0.0 & 1.0 & 0.0 & 0.0 & 0.0 & 1.0 & 1.0000 & 0.0000 & 2.0000 \\
\hline 25 & 1.0 & 0.0 & 0.0 & 0.0 & 1.0 & 0.0 & 0.0 & 0.0 & 1.0 & 00 & 1.0000 & 1.0000 \\
\hline 26 & 1.0 & 0.0 & 0.0 & 0.0 & .0 & 0.0 & 0.0 & 0.0 & 1.0 & 00 & 00 & 2.0000 \\
\hline 27 & -1.0 & 0.0 & 0.0 & 0.0 & 1.0 & 0.0 & 0.0 & 0.0 & -1.0 & 0.0000 & -1 & -0.5000 \\
\hline 28 & -1.0 & 0.0 & 0.0 & 0.0 & 1.0 & 0.0 & 0.0 & 0.0 & -1.0 & 00 & -1 & 0.5000 \\
\hline 29 & -1.0 & 0.0 & 0.0 & 0.0 & 1.0 & 0.0 & 0.0 & 0.0 & -1.0 & 0.0000 & 00 & -0.5000 \\
\hline 30 & -1.0 & 0.0 & 0.0 & 0.0 & 1.0 & 0.0 & 0.0 & 0.0 & -1.0 & 00 & 1. & -0.5000 \\
\hline 31 & -1.0 & 0.0 & 0.0 & 0.0 & 1.0 & 0.0 & 0.0 & 0.0 & -1.0 & 0 . & 1. & 0.5000 \\
\hline 32 & -1.0 & 0.0 & 0.0 & 0.0 & 1.0 & 0.0 & 0.0 & 0.0 & -1.0 & 00 & -1. & 0.5000 \\
\hline 33 & -1.0 & 0.0 & 0.0 & 0.0 & 1.0 & 0.0 & 0.0 & 0.0 & -1.0 & 1.0000 & -1.0000 & 1.5000 \\
\hline 34 & -1.0 & 0.0 & 0.0 & 0.0 & 1.0 & 0.0 & 0.0 & 0.0 & -1.0 & 000 & 0.0000 & -0.5000 \\
\hline 35 & -1.0 & 0.0 & 0.0 & 0.0 & 1.0 & 0.0 & 0.0 & 0.0 & -1.0 & 1. & 0. & 0 . \\
\hline 36 & -1.0 & 0.0 & 0.0 & 0.0 & 1.0 & 0.0 & 0.0 & 0.0 & -1.0 & 1.0000 & 00 & 1.5000 \\
\hline 37 & -1.0 & 0.0 & 0.0 & 0.0 & 1.0 & 0.0 & 0.0 & 0.0 & -1.0 & 00 & 000 & 2.5000 \\
\hline 38 & -1.0 & 0.0 & 0.0 & 0 . & 1.0 & 0.0 & 0.0 & 0.0 & -1.0 & & & 0 . \\
\hline 39 & -1.0 & 0.0 & 0.0 & 0.0 & 1.0 & 0.0 & 0.0 & 0.0 & -1.0 & 00 & 1. & 1. \\
\hline 40 & -1.0 & 0.0 & 0.0 & 0.0 & 1.0 & 0.0 & 0.0 & 0.0 & -1.0 & 00 & -1.0000 & 1.5000 \\
\hline 41 & -1.0 & 0.0 & 0.0 & 0.0 & 1.0 & 0. & 0.0 & 0.0 & -1.0 & & -1. & 2.5000 \\
\hline 42 & -1.0 & 0.0 & 0.0 & 0.0 & 1.0 & 0.0 & 0.0 & 0.0 & -1.0 & 00 & 00 & 1. \\
\hline 43 & -1.0 & 0.0 & 0.0 & 0.0 & 1.0 & 0.0 & 0.0 & 0.0 & -1.0 & 2.0000 & 0.0000 & 2.5000 \\
\hline 44 & -1.0 & 0.0 & 0.0 & 0.0 & 1.0 & 0 . & 0 . & 0.0 & -1.0 & & & \\
\hline 45 & -1.0 & 0.0 & 0 . & 0.0 & 1.0 & 0.0 & 0.0 & 0.0 & -1.0 & 00 & 1. & 00 \\
\hline 46 & 1.0 & 0.0 & 0.0 & 0.0 & 1.0 & 0.0 & 0.0 & 0.0 & 1.0 & -1.5000 & -0.5000 & -2.0000 \\
\hline 47 & 1.0 & 0.0 & 0.0 & 0. & 1.0 & 0. & 0 . & 0.0 & 1.0 & -1 & 00 & -2.0000 \\
\hline 48 & 1.0 & 0.0 & 0 . & 0. & 1.0 & 0 & 0 & 0.0 & 1.0 & -0 . & -1 & -1 \\
\hline 49 & 1.0 & 0.0 & 0.0 & 0. & 1.0 & 0.0 & 0.0 & 0.0 & 1.0 & -0. & -1.5000 & 0.0000 \\
\hline 50 & 1.0 & 0.0 & 0.0 & 0. & 1.0 & 0. & 0.0 & 0.0 & 1.0 & -0 . & -0 . & -2.0000 \\
\hline 51 & 1.0 & 0.6 & 0. & 0 . & 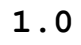 & & 0 & 0.0 & 1.0 & -0 & & $-1.0 c$ \\
\hline 52 & 1.0 & 0.0 & 0 . & 0. & 1.0 & 0.0 & 0 . & 0.0 & 1.0 & -0. & -0.5000 & 0.0000 \\
\hline 53 & 1.0 & 0.0 & 0.0 & 0. & 1.0 & 0.0 & 0.0 & 0.0 & 1.0 & -0.5000 & -0.5000 & 1.0000 \\
\hline 54 & 1.0 & 0.0 & 0.0 & u. & 1.0 & 0. & 0.0 & 0.0 & 1.0 & -0 . & 00 & -2.0000 \\
\hline 55 & 1.0 & 0.0 & 0 . & 0 . & 1.0 & 0 . & 0.0 & 0.0 & 1.0 & -0 . & 00 & -1.0000 \\
\hline 56 & 1.0 & 0.0 & 0.0 & 0.0 & 1.0 & 0.0 & 0.0 & 0.0 & 1.0 & -0.5000 & 0.5000 & 0.0000 \\
\hline 57 & 1.0 & 0.0 & 0.0 & 0.0 & 1.0 & 0.0 & 0.0 & 0.0 & 1.0 & -0.5000 & 0.5000 & 1.0000 \\
\hline 58 & 1.0 & 0.0 & 0 . & 0 . & 1.0 & 0. & 0.0 & 0.0 & 1.0 & -0.5000 & 1.5000 & -1.0000 \\
\hline 59 & 1.0 & 0.0 & 0.0 & 0.0 & 1.0 & 0.0 & 0.0 & 0.0 & 1.0 & -0.5000 & 1.5000 & 0.0000 \\
\hline 60 & 1.0 & 0.0 & 0. & 0.0 & 1.0 & 0. & 0.0 & 0.0 & 1.0 & & -1.5000 & \\
\hline 61 & 1.0 & 0.0 & 0.0 & 0.0 & 1.0 & 0.0 & 0.0 & 0.0 & 1.0 & 0.5000 & -1.5000 & 1.0000 \\
\hline
\end{tabular}




\begin{tabular}{|c|c|c|c|c|c|c|c|c|c|c|c|c|}
\hline 62 & 1.0 & 0.0 & 0.0 & 0.0 & 1.0 & 0.0 & 0.0 & 0.0 & 1.0 & 0.5000 & -0.5000 & -1.0000 \\
\hline 63 & 1.0 & 0.0 & 0.0 & 0.0 & 1.0 & 0.0 & 0.0 & 0.0 & 1.0 & 0.5000 & -0.5000 & 0.0000 \\
\hline 64 & 1.0 & 0.0 & 0.0 & 0.0 & 1.0 & 0.0 & 0.0 & 0.0 & 1.0 & .5000 & -0.5000 & 1.0000 \\
\hline 65 & 1.0 & 0.0 & 0.0 & 0.0 & 1.0 & 0.0 & 0.0 & 0.0 & 1.0 & 0.5000 & -0.5000 & 2.0000 \\
\hline 66 & 1.0 & 0.0 & 0.0 & 0.0 & 1.0 & 0.0 & 0.0 & 0.0 & 1.0 & 0.5000 & 0.5000 & -1.0000 \\
\hline 67 & 1.0 & 0.0 & 0.0 & 0.0 & 1.0 & 0.0 & 0.0 & 0.0 & 1.0 & & 0.5000 & 1.0000 \\
\hline 68 & 1.0 & 0.0 & 0.0 & 0.0 & 1.0 & 0.0 & 0.0 & 0.0 & 1.0 & 0.5000 & 0.5000 & 2.0000 \\
\hline 69 & 1.0 & 0.0 & 0.0 & 0.0 & 1.0 & 0.0 & 0.0 & 0.0 & 1.0 & 0.5000 & 1.5000 & 0.0000 \\
\hline 70 & 1.0 & 0.0 & 0.0 & 0.0 & 1.0 & 0.0 & 0.0 & 0.0 & 1.0 & 0.5000 & .5000 & 1.0000 \\
\hline 71 & 1.0 & 0.0 & 0.0 & 0.0 & 1.0 & 0.0 & 0.0 & 0.0 & 1.0 & 1.5000 & -0.5000 & 2.0000 \\
\hline 72 & 1.0 & 0.0 & 0.0 & 0.0 & 1.0 & 0.0 & 0.0 & 0.0 & 1.0 & 1.5000 & 0.5000 & 2.0000 \\
\hline 73 & -1.0 & 0.0 & 0.0 & 0.0 & 1.0 & 0.0 & 0.0 & 0.0 & -1.0 & -0.5000 & -0.5000 & -0.5000 \\
\hline 74 & -1.0 & 0.0 & 0.0 & 0.0 & 1.0 & 0.0 & 0.0 & 0.0 & -1.0 & -0.5000 & 0.5000 & -0.5000 \\
\hline 75 & -1.0 & 0.0 & 0.0 & 0.0 & 1.0 & 0.0 & 0.0 & 0.0 & -1.0 & 0.5000 & -1.5000 & 0.5000 \\
\hline 76 & -1.0 & 0.0 & 0.0 & 0.0 & 1.0 & 0.0 & 0.0 & 0.0 & -1.0 & 000 & -0.5000 & -0.5000 \\
\hline 77 & -1.0 & 0.0 & 0.0 & 0.0 & 1.0 & 0.0 & 0.0 & 0.0 & -1.0 & 0.5000 & -0.5000 & 0.5000 \\
\hline 78 & -1.0 & 0.0 & 0.0 & 0.0 & 1.0 & 0.0 & 0.0 & 0.0 & -1.0 & 000 & -0.5000 & 1.5000 \\
\hline 79 & -1.0 & 0.0 & 0.0 & 0.0 & 1.0 & 0.0 & 0.0 & 0.0 & -1.0 & 0.5000 & 0.5000 & -0.5000 \\
\hline 80 & -1.0 & 0.0 & 0.0 & 0.0 & 1.0 & 0.0 & 0.0 & 0.0 & -1.0 & 0.5000 & 0.5000 & 1.5000 \\
\hline 81 & -1.0 & 0.0 & 0.0 & 0.0 & 1.0 & 0.0 & 0.0 & 0.0 & -1.0 & 0.5000 & 1.5000 & 0.5000 \\
\hline 82 & -1.0 & 0.0 & 0.0 & 0.0 & 1.0 & 0.0 & 0.0 & 0.0 & -1.0 & 00 & -1 & 1.5000 \\
\hline 83 & -1.0 & 0.0 & 0.0 & 0.0 & 1.0 & 0.0 & 0.0 & 0.0 & -1.0 & 1.5000 & -0.5000 & 0.5000 \\
\hline 84 & -1.0 & 0.0 & 0.0 & 0.0 & 1.0 & 0.0 & 0.0 & 0.0 & -1.0 & 1.5000 & -0.5000 & 1.5000 \\
\hline 85 & -1.0 & 0.0 & 0.0 & 0 . & 1.0 & 0.0 & 0.0 & 0.0 & -1.0 & 00 & -0.5000 & 2.5000 \\
\hline 86 & -1.0 & 0.0 & 0.0 & 0.0 & 1.0 & 0.0 & 0.0 & 0.0 & -1.0 & 1.5000 & 0.5000 & 0.5000 \\
\hline 87 & -1.0 & 0.0 & 0.0 & 0.0 & 1.0 & 0.0 & 0.0 & 0.0 & -1.0 & 1.5000 & 0.5000 & 1.5000 \\
\hline 88 & -1.0 & 0.0 & 0.0 & 0.0 & 1.0 & 0.0 & 0.0 & 0.0 & -1.0 & 1.5000 & 0.5000 & 2.5000 \\
\hline 89 & -1.0 & 0.0 & 0.0 & 0.0 & 1.0 & 0.0 & 0.0 & 0.0 & -1.0 & 1.5000 & 1.5000 & 1.5000 \\
\hline 90 & -1.0 & 0.0 & 0.0 & 0.0 & 1.0 & 0.0 & 0.0 & 0.0 & -1.0 & 2.5000 & -0.5000 & 2.5000 \\
\hline 91 & -1.0 & 0.0 & 0.0 & 0.0 & 1.0 & 0.0 & 0.0 & 0.0 & -1.0 & 2.5000 & 0.5000 & 2.5000 \\
\hline-2 & .1 & 10.0 & & & 78 & & 6.1 & -32 & 2.6 & -4.2 & -36.8 & \\
\hline
\end{tabular}

a...a energies

$\begin{array}{rrrrrrrrrr}1 & 2 & 10.083 & 0.1 & -0.0 & -0.2 & 0.0 & -0.1 & -0.4 & 0.2 \\ 1 & 3 & 7.284 & -0.2 & -0.1 & -5.6 & 3.7 & -2.1 & 1.2 & 2.1 \\ 1 & 4 & 5.695 & -6.9 & -1.8 & -14.2 & 13.9 & -9.0 & -3.7 & -7.2 \\ 1 & 5 & 13.414 & 0.0 & -0.0 & -0.0 & 0.0 & -0.0 & -0.1 & -0.0 \\ 1 & 6 & 11.389 & -0.0 & -0.0 & -0.1 & 0.0 & -0.1 & -0.2 & -0.1 \\ 1 & 7 & 11.279 & 0.1 & -0.0 & -0.0 & 0.0 & 0.1 & -0.1 & 0.3 \\ 1 & 8 & 8.776 & -0.2 & -0.0 & -0.3 & 0.0 & -0.5 & -0.7 & -0.7 \\ 1 & 9 & 12.630 & 0.0 & -0.0 & -0.0 & 0.0 & 0.0 & -0.1 & 0.1 \\ 1 & 10 & 13.414 & 0.0 & -0.0 & -0.0 & 0.0 & -0.0 & -0.1 & 0.0 \\ 1 & 11 & 11.389 & -0.1 & -0.0 & -0.1 & 0.0 & -0.1 & -0.2 & -0.1 \\ 1 & 12 & 10.912 & -0.1 & -0.0 & -0.1 & 0.0 & -0.1 & -0.2 & -0.2 \\ 1 & 13 & 7.260 & -0.4 & -0.1 & -6.3 & 5.1 & -1.7 & 2.8 & 2.4 \\ 1 & 14 & 10.912 & -0.0 & -0.0 & -0.1 & 0.0 & -0.1 & -0.2 & -0.2 \\ 1 & 15 & 8.146 & -0.2 & -0.0 & -0.3 & 0.0 & -0.5 & -0.8 & -0.5 \\ 1 & 16 & 8.146 & -0.2 & -0.0 & -0.3 & 0.0 & -0.5 & -0.8 & -0.5 \\ 1 & 17 & 10.912 & -0.0 & -0.0 & -0.1 & 0.0 & -0.1 & -0.2 & -0.2 \\ 1 & 18 & 7.260 & -0.4 & -0.1 & -6.3 & 5.1 & -1.7 & 2.8 & 2.4 \\ 1 & 19 & 10.912 & -0.1 & -0.0 & -0.1 & 0.0 & -0.1 & -0.2 & -0.2 \\ 1 & 20 & 11.389 & -0.1 & -0.0 & -0.1 & 0.0 & -0.1 & -0.2 & -0.1 \\ 1 & 21 & 13.414 & 0.0 & -0.0 & -0.0 & 0.0 & -0.0 & -0.1 & 0.0 \\ 1 & 22 & 12.630 & 0.0 & -0.0 & -0.0 & 0.0 & 0.0 & -0.1 & 0.1 \\ 1 & 23 & 8.776 & -0.2 & -0.0 & -0.3 & 0.0 & -0.5 & -0.7 & -0.7 \\ 1 & 24 & 11.279 & 0.1 & -0.0 & -0.0 & 0.0 & 0.1 & -0.1 & 0.3 \\ 1 & 25 & 11.389 & -0.0 & -0.0 & -0.1 & 0.0 & -0.1 & -0.2 & -0.1 \\ 1 & 26 & 13.414 & 0.0 & -0.0 & -0.0 & 0.0 & -0.0 & -0.1 & -0.0 \\ 1 & 27 & 11.757 & -0.0 & -0.0 & -0.0 & 0.0 & -0.1 & -0.1 & -0.1 \\ 1 & 28 & 12.425 & 0.0 & -0.0 & -0.0 & 0.0 & -0.0 & -0.1 & 0.1 \\ 1 & 29 & 9.248 & 0.1 & -0.0 & -0.1 & 0.0 & -0.1 & -0.3 & 0.2 \\ 1 & 30 & 11.757 & -0.0 & -0.0 & -0.0 & 0.0 & -0.1 & -0.1 & -0.1\end{array}$




\begin{tabular}{|c|c|c|c|c|c|c|c|c|c|}
\hline 1 & 31 & 12.425 & 0.0 & -0.0 & -0.0 & 0.0 & -0.0 & -0.1 & 0.1 \\
\hline 1 & 32 & 8.325 & -0.1 & -0.0 & -0.8 & 0.0 & -0.9 & -1.4 & -0.5 \\
\hline 1 & 33 & 8.325 & -0.1 & -0.0 & -0.8 & 0.0 & -0.9 & -1.4 & -0.5 \\
\hline 1 & 34 & 12.219 & -0.0 & -0.0 & -0.0 & 0.0 & -0.1 & -0.1 & -0.1 \\
\hline 1 & 35 & 4.073 & -10.0 & -7.1 & -28.8 & 37.9 & -7.9 & -17.7 & 4.0 \\
\hline 1 & 36 & 4.073 & -10.0 & -7.1 & -28.8 & 37.5 & -8.4 & -17.7 & 4.0 \\
\hline 1 & 37 & 12.219 & -0.0 & -0.0 & -0.0 & 0.0 & -0.1 & -0.1 & -0.1 \\
\hline 1 & 38 & 8.325 & -0.1 & -0.0 & -0.8 & 0.0 & -0.9 & -1.4 & -0.5 \\
\hline 1 & 39 & 8.325 & -0.1 & -0.0 & -0.8 & 0.0 & -0.9 & -1.4 & -0.5 \\
\hline 1 & 40 & 12.425 & 0.0 & -0.0 & -0.0 & 0.0 & -0.0 & -0.1 & 0.1 \\
\hline 1 & 41 & 11.757 & -0.0 & -0.0 & -0.0 & 0.0 & -0.1 & -0.1 & -0.1 \\
\hline 1 & 42 & 10.083 & 0.1 & -0.0 & -0.2 & 0.0 & -0.1 & -0.4 & 0.2 \\
\hline 1 & 43 & 9.248 & 0.1 & -0.0 & -0.1 & 0.0 & -0.1 & -0.3 & 0.2 \\
\hline 1 & 44 & 12.425 & 0.0 & -0.0 & -0.0 & 0.0 & -0.0 & -0.1 & 0.1 \\
\hline 1 & 45 & 11.757 & -0.0 & -0.0 & -0.0 & 0.0 & -0.1 & -0.1 & -0.1 \\
\hline 1 & 46 & 13.818 & -0.0 & -0.0 & -0.0 & 0.0 & -0.0 & -0.0 & -0.0 \\
\hline 1 & 47 & 13.818 & -0.0 & -0.0 & -0.0 & 0.0 & -0.0 & -0.0 & -0.0 \\
\hline 1 & 48 & 12.264 & -0.0 & -0.0 & -0.0 & 0.0 & -0.1 & -0.1 & -0.1 \\
\hline 1 & 49 & 12.589 & 0.0 & -0.0 & -0.0 & 0.0 & -0.0 & -0.1 & 0.1 \\
\hline 1 & 50 & 13.024 & 0.0 & -0.0 & -0.0 & 0.0 & -0.0 & -0.1 & 0.0 \\
\hline 1 & 51 & 6.707 & 0.3 & -0.0 & -0.9 & 0.0 & -0.7 & -2.0 & 0.4 \\
\hline 1 & 52 & 7.284 & -0.2 & -0.1 & -5.6 & 3.7 & -2.1 & 1.2 & 2.1 \\
\hline 1 & 53 & 13.923 & -0.0 & -0.0 & -0.0 & 0.0 & -0.0 & -0.1 & -0.0 \\
\hline 1 & 54 & 13.024 & 0.0 & -0.0 & -0.0 & 0.0 & 0.0 & -0.1 & 0.0 \\
\hline 1 & 55 & 6.707 & 0.3 & -0.0 & -0.9 & 0.0 & -0.7 & -1.9 & 0.8 \\
\hline 1 & 56 & 7.284 & 0.3 & -0.1 & -5.7 & 4.0 & -1.5 & 1.5 & 2.3 \\
\hline 1 & 57 & 13.923 & -0.0 & -0.0 & -0.0 & 0.0 & -0.0 & -0.1 & -0.0 \\
\hline 1 & 58 & 12.264 & -0.0 & -0.0 & -0.0 & 0.0 & -0.1 & -0.1 & -0.1 \\
\hline 1 & 59 & 12.589 & 0.0 & -0.0 & -0.0 & 0.0 & 0.0 & -0.1 & 0.1 \\
\hline 1 & 60 & 12.589 & 0.0 & -0.0 & -0.0 & 0.0 & 0.0 & -0.1 & 0.1 \\
\hline 1 & 61 & 12.264 & -0.0 & -0.0 & -0.0 & 0.0 & -0.1 & -0.1 & -0.1 \\
\hline 1 & 62 & 13.923 & -0.0 & -0.0 & -0.0 & 0.0 & -0.0 & -0.1 & -0.0 \\
\hline 1 & 63 & 7.284 & 0.3 & -0.1 & -5.7 & 4.0 & -1.5 & 1.5 & 2.3 \\
\hline 1 & 64 & 6.707 & 0.3 & -0.0 & -0.9 & 0.0 & -0.7 & -1.9 & 0.8 \\
\hline 1 & 65 & 13.024 & 0.0 & -0.0 & -0.0 & 0.0 & 0.0 & -0.1 & 0.0 \\
\hline 1 & 66 & 13.923 & -0.0 & -0.0 & -0.0 & 0.0 & -0.0 & -0.1 & -0.0 \\
\hline 1 & 67 & 6.707 & 0.3 & -0.0 & -0.9 & 0.0 & -0.7 & -2.0 & 0.4 \\
\hline 1 & 68 & 13.024 & 0.0 & -0.0 & -0.0 & 0.0 & -0.0 & -0.1 & 0.0 \\
\hline 1 & 69 & 12.589 & 0.0 & -0.0 & -0.0 & 0.0 & -0.0 & -0.1 & 0.1 \\
\hline 1 & 70 & 12.264 & -0.0 & -0.0 & -0.0 & 0.0 & -0.1 & -0.1 & -0.1 \\
\hline 1 & 71 & 13.818 & -0.0 & -0.0 & -0.0 & 0.0 & -0.0 & -0.0 & -0.0 \\
\hline 1 & 72 & 13.818 & -0.0 & -0.0 & -0.0 & 0.0 & -0.0 & -0.0 & -0.0 \\
\hline 1 & 73 & 13.655 & -0.0 & -0.0 & -0.0 & 0.0 & -0.0 & -0.1 & -0.1 \\
\hline 1 & 74 & 13.655 & -0.0 & -0.0 & -0.0 & 0.0 & -0.0 & -0.1 & -0.1 \\
\hline 1 & 75 & 11.741 & 0.0 & -0.0 & -0.1 & 0.0 & -0.0 & -0.2 & 0.1 \\
\hline 1 & 76 & 9.524 & 0.1 & -0.0 & -0.1 & 0.0 & -0.0 & -0.3 & 0.3 \\
\hline 1 & 77 & 5.695 & -6.9 & -1.8 & -14.2 & 13.9 & -8.9 & -3.7 & -7.2 \\
\hline 1 & 78 & 10.337 & -0.0 & -0.0 & -0.1 & 0.0 & -0.1 & -0.3 & -0.0 \\
\hline 1 & 79 & 9.524 & 0.1 & -0.0 & -0.1 & 0.0 & -0.0 & -0.3 & 0.3 \\
\hline 1 & 80 & 10.337 & -0.0 & -0.0 & -0.1 & 0.0 & -0.1 & -0.3 & -0.0 \\
\hline 1 & 81 & 11.741 & 0.0 & -0.0 & -0.1 & 0.0 & -0.0 & -0.2 & 0.1 \\
\hline 1 & 82 & 11.741 & 0.0 & -0.0 & -0.1 & 0.0 & -0.0 & -0.2 & 0.1 \\
\hline 1 & 83 & 10.337 & -0.0 & -0.0 & -0.1 & 0.0 & -0.1 & -0.3 & -0.0 \\
\hline 1 & 84 & 5.695 & -6.9 & -1.8 & -14.2 & 13.7 & -9.1 & -3.7 & -7.2 \\
\hline 1 & 85 & 9.524 & 0.1 & -0.0 & -0.1 & 0.0 & -0.0 & -0.3 & 0.3 \\
\hline 1 & 86 & 10.337 & -0.0 & -0.0 & -0.1 & 0.0 & -0.1 & -0.3 & -0.0 \\
\hline 1 & 87 & 5.695 & -6.9 & -1.8 & -14.2 & 13.7 & -9.1 & -3.7 & -7.2 \\
\hline 1 & 88 & 9.524 & 0.1 & -0.0 & -0.1 & 0.0 & -0.0 & -0.3 & 0.3 \\
\hline 1 & 89 & 11.741 & 0.0 & -0.0 & -0.1 & 0.0 & -0.0 & -0.2 & 0.1 \\
\hline 1 & 90 & 13.655 & -0.0 & -0.0 & -0.0 & 0.0 & -0.0 & -0.1 & -0.1 \\
\hline 1 & 91 & 13.655 & -0.0 & -0.0 & -0.0 & 0.0 & -0.0 & -0.1 & -0.1 \\
\hline
\end{tabular}




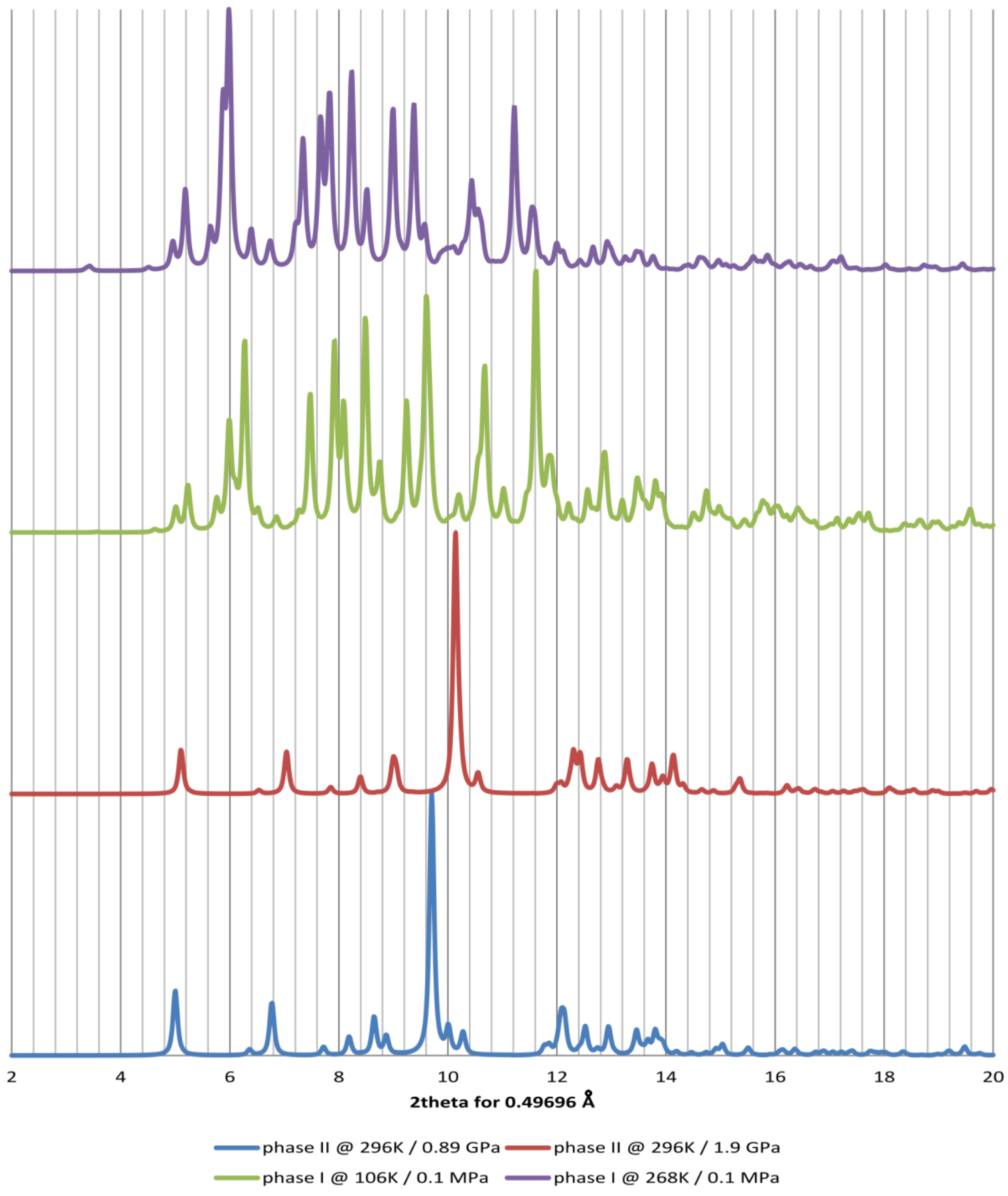

Figure S3. Powder-Diffraction patterns based on the $\mathrm{C}_{6} \mathrm{~F}_{6}$ phases I and II, calculated for $\lambda=0.49696 \AA$, for convenient comparison with Figure 7 in Reference 59. 